\title{
Sensitivity of Wind Turbine Airfoil Sections to Geometry Variations Inherent in Modular Blades
}

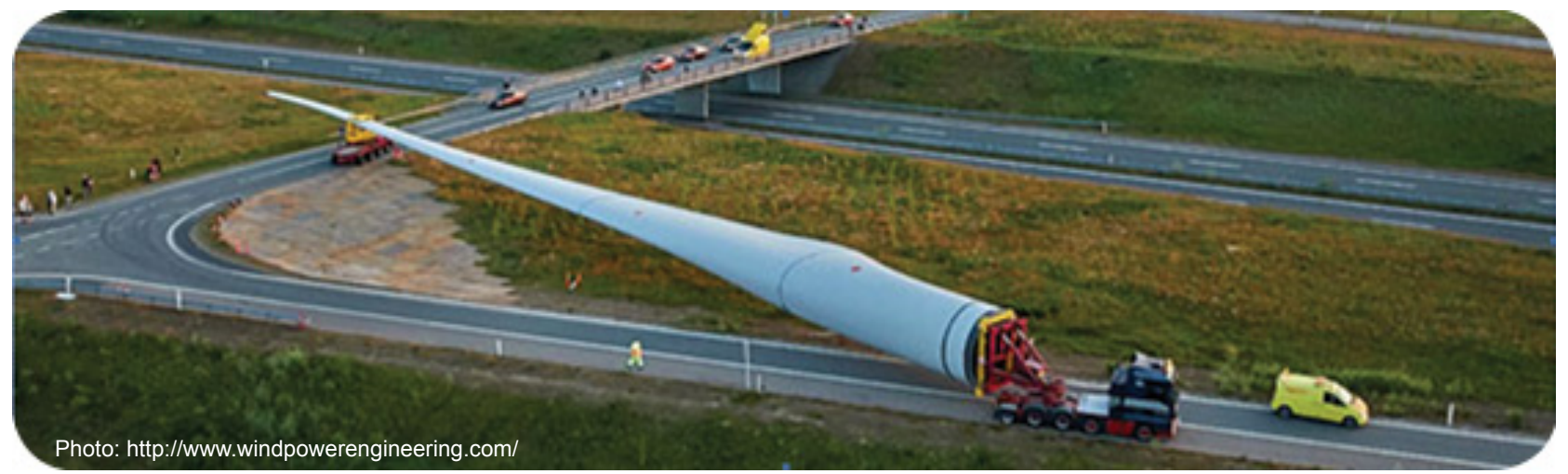

Ken Brown, Nick Molinaro, Tim Meyers, Mike Nelson, Aurelien Borgoltz, Nanya Intaratep, William Devenport

Virginia Tech, Blacksburg, VA, U.S.A.

\section{Jonathan Luedke, David Pesetsky}

GE Power and Water, Greenville, SC, U.S.A.

June 9, 2015 NAWEA Symposium
The information, data, or work presented herein was funded in part by the Advanced Research Projects Agency-Energy (ARPA-E), U.S.

Department of Energy, under Award Number DE-AR0000293.

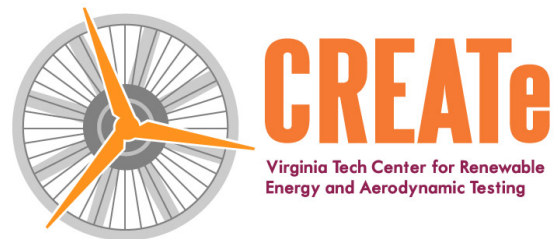




\section{Motivation}

Wind turbine blades need to grow longer to lower the cost of electricity

\section{Conventional}

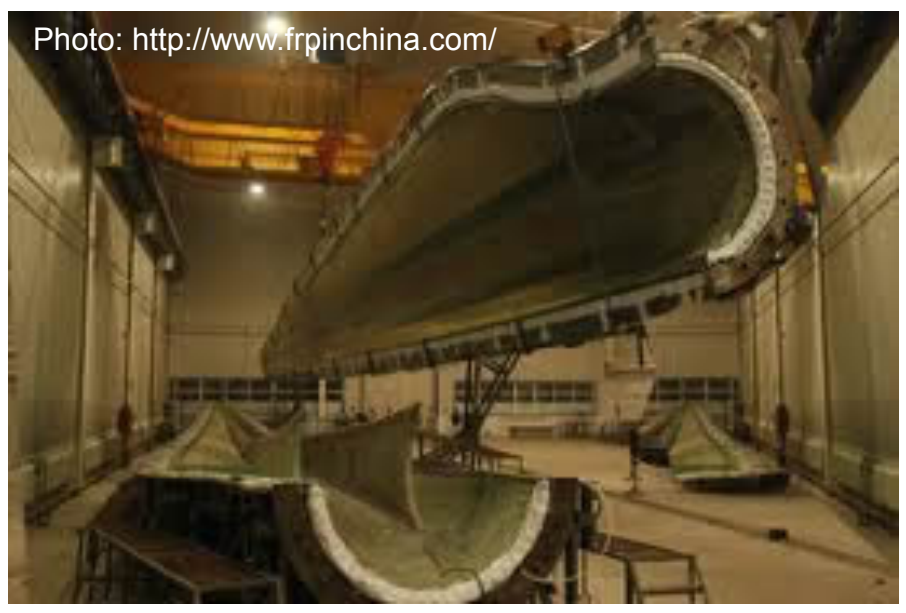

\section{Modular}

Photo: courtesy of GE Power and Water

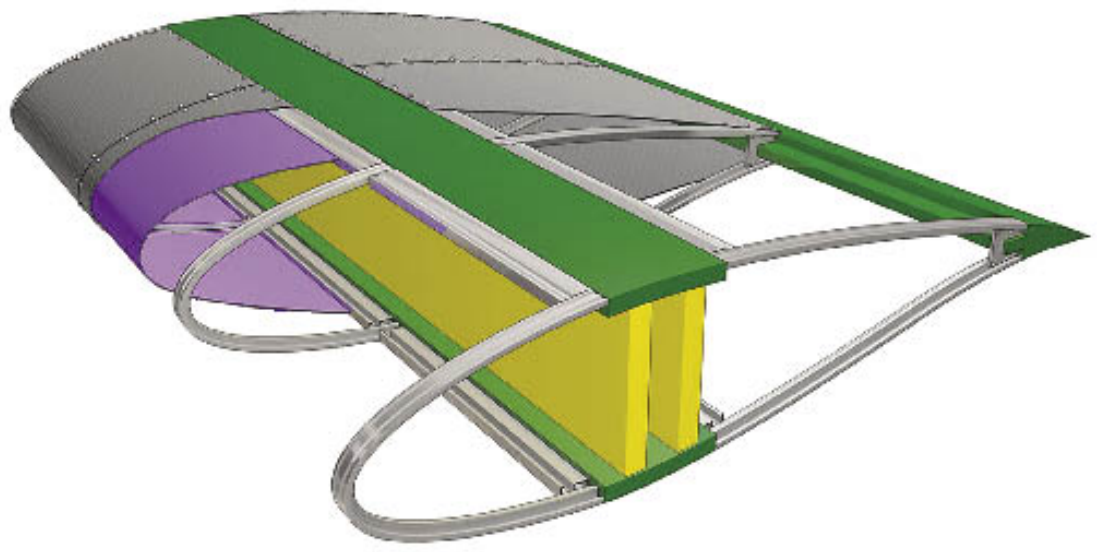

2-piece mold shell manufacturing $\longrightarrow$ Modular manufacturing Transportation difficulties Build on-site

Low stiffness/weight ratio Tailor-able stiffness/weight ratio

Fixed outer mold line Misaligned/flexible outer mold line Dutton et al. (2000), Vionis et al. (2006), Saenz et al. (2014) 


\section{Outline}

\section{Modular wind turbine blades}

Misaligned sections

- Literature

- Configuration

- Test setup

- Results

\section{Fabric sections}

- Literature

- Configuration

- Test setup

- Results

\section{Summary}




\section{Misaligned sections: literature}

\section{Transition behavior}

2D disturbances thought to contribute to Tollmein-Schlichting type transition (Braslow, 1960)

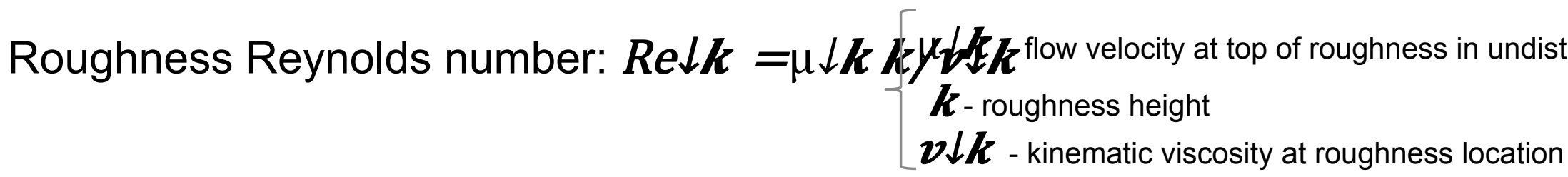

\begin{tabular}{|l|l|l|}
\hline Study & Geometry & Re $\downarrow k$, critical \\
\hline Fage (1943) & Flat/arched ridge & $204-240$ \\
\hline Smith and Clutter (1959) & Spanwise wires & $40-260$ \\
\hline Drake et al. (2010) & Forward-facing step & $150-2,100$ \\
\hline Drake et al. (2010) & Backward-facing step & $75-800$ \\
\hline Current study & Backward- and forward-facing ramp & $?$
\end{tabular}

Re $\downarrow k$,critical may be influenced by length of preceding laminar boundary layer, width of geometric feature, type of the geometric feature, and pressure gradient in boundary layer 


\section{Misaligned sections: configuration}

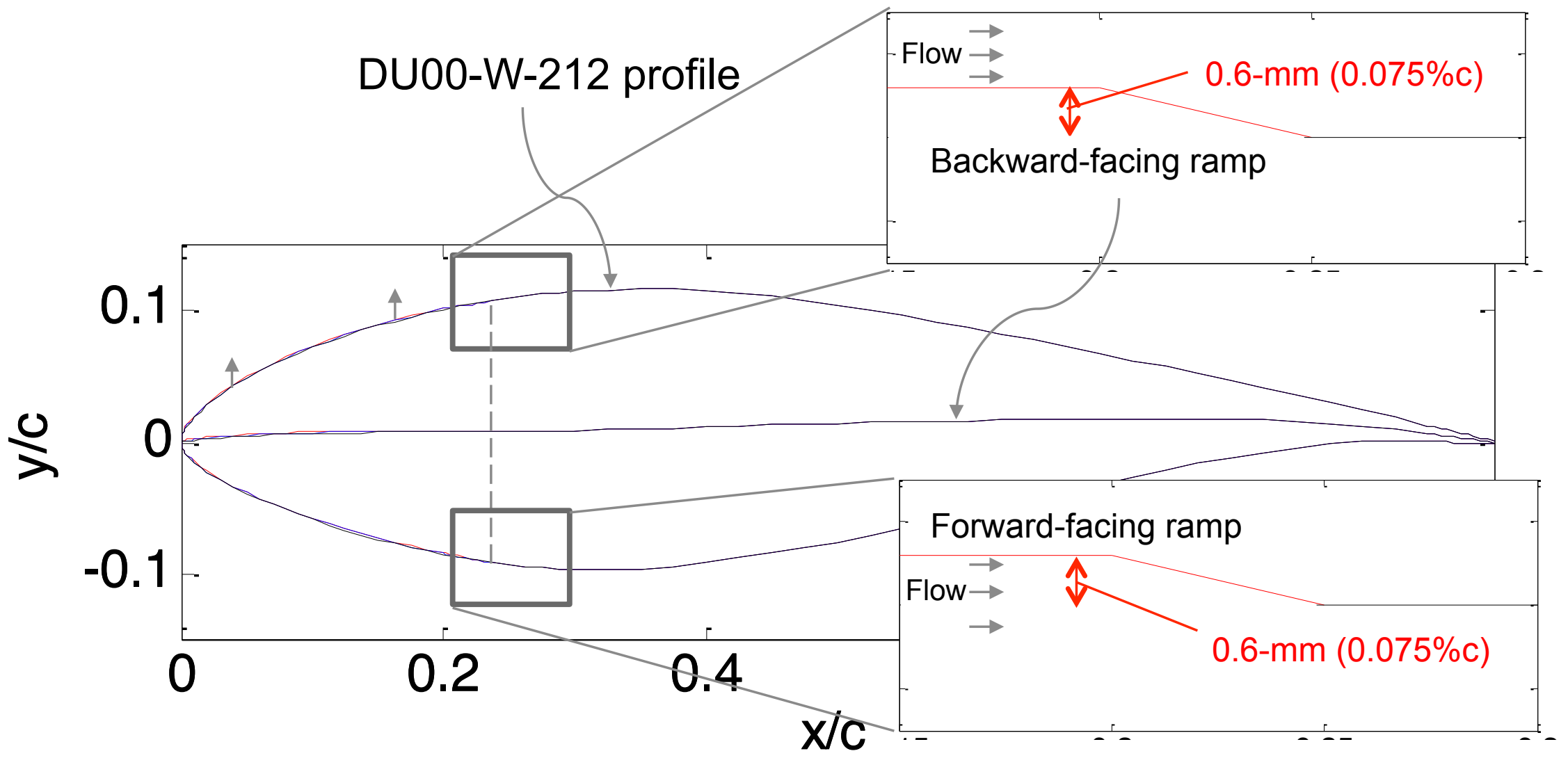

Offset results in an effective increase in camber and, more importantly, produce discontinuities in the profile 


\section{Misaligned sections: test setup}

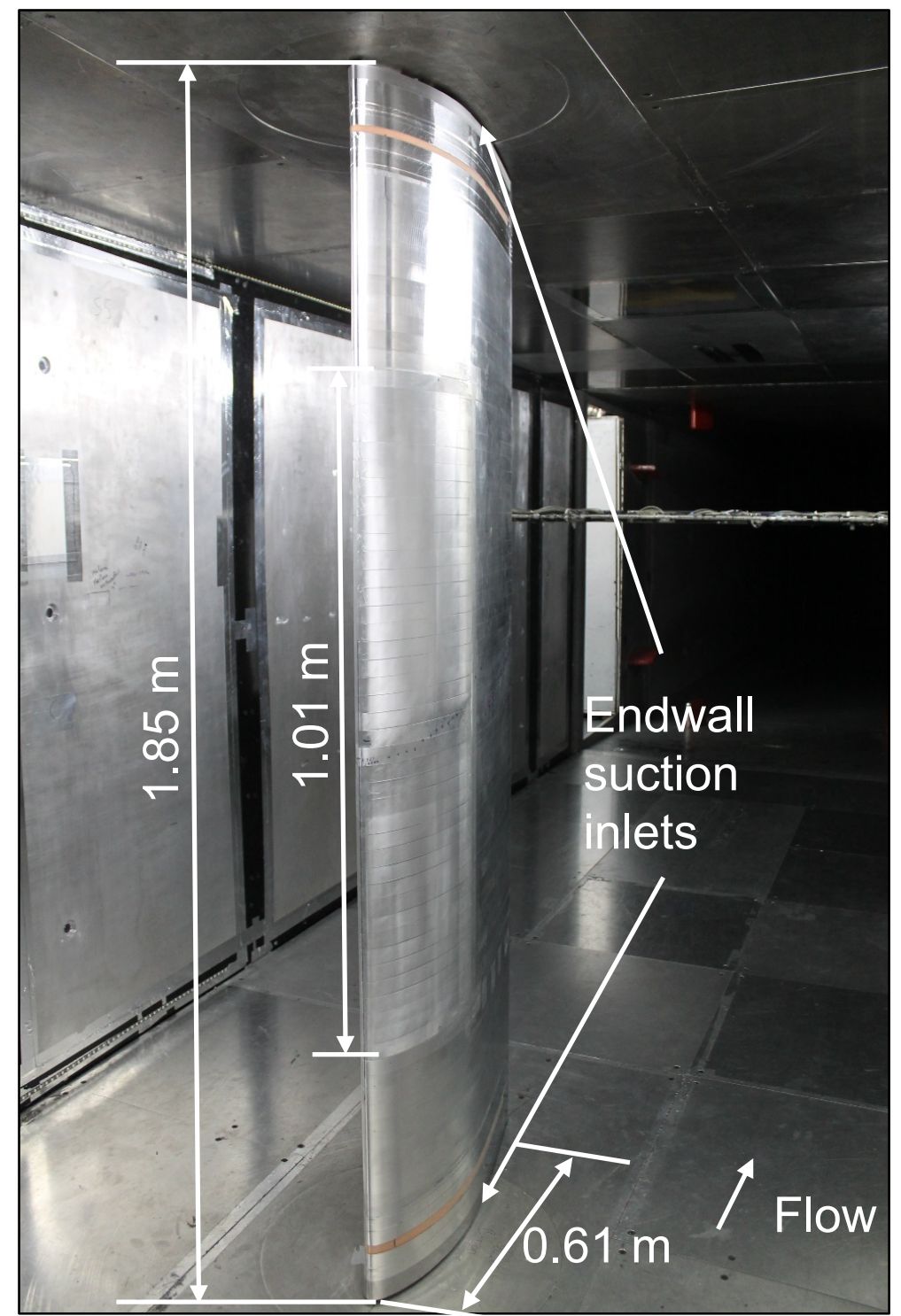

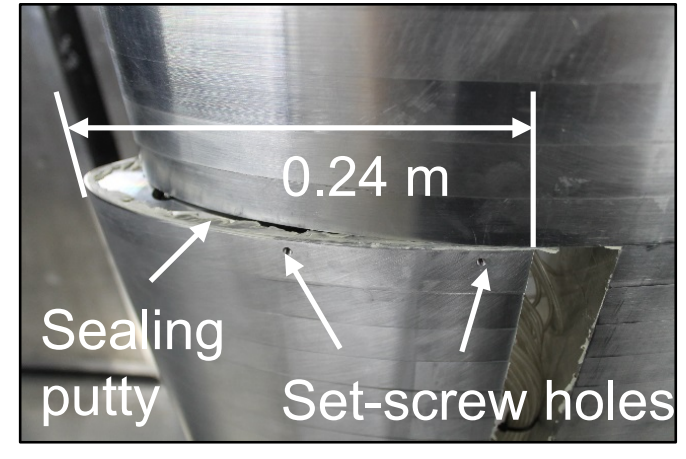

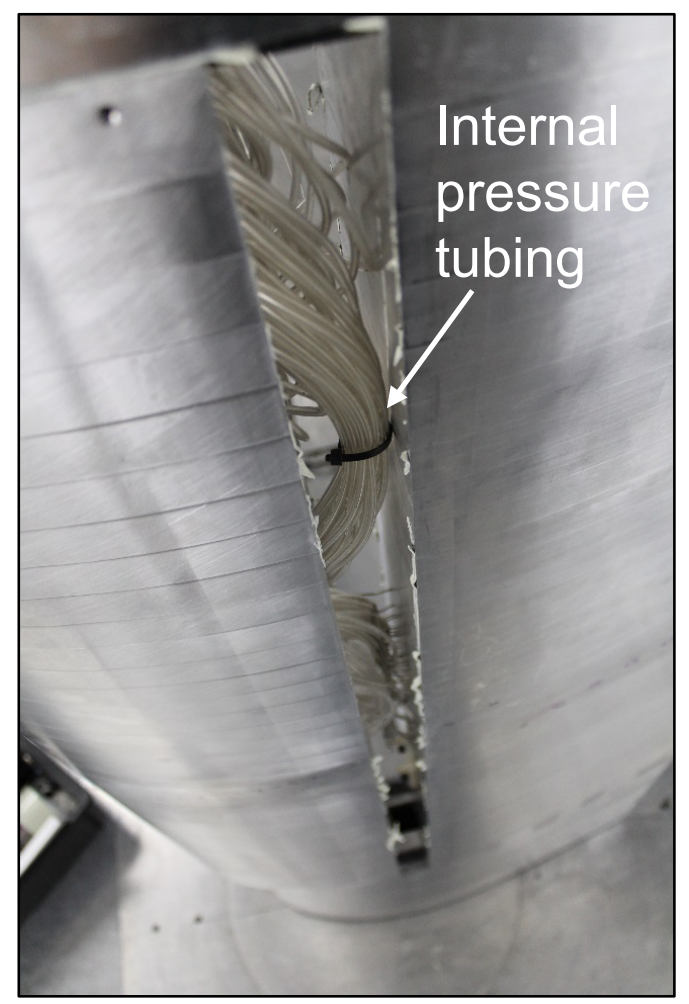

Model:

- DU00-W-212

- 0.6-m chord

- 64 aluminum laminates

- 73 pressure taps

- 3 nose modules: baseline, 0.3-, and 0.6-mm offsets

- Endwall suction on

Measurements:

- Mean surface pressure

- Wake profiles

Test Conditions:

- $\operatorname{Re}=2.5 \times 10^{6}$

- $\mathrm{Ma}=0.21$ 


\section{Misaligned sections: results}
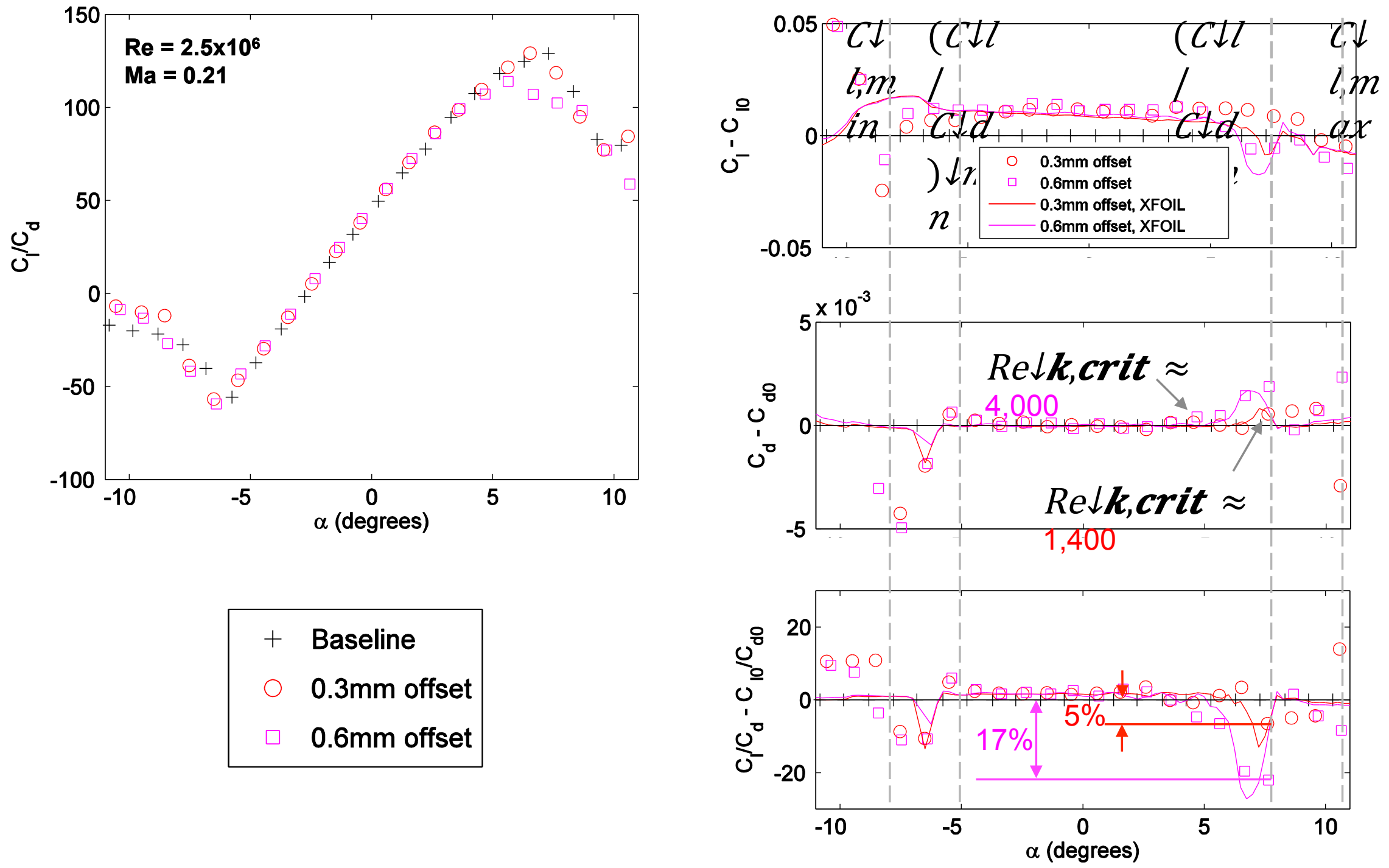


\section{Misaligned sections: results}

\section{XFOIL transition prediction}

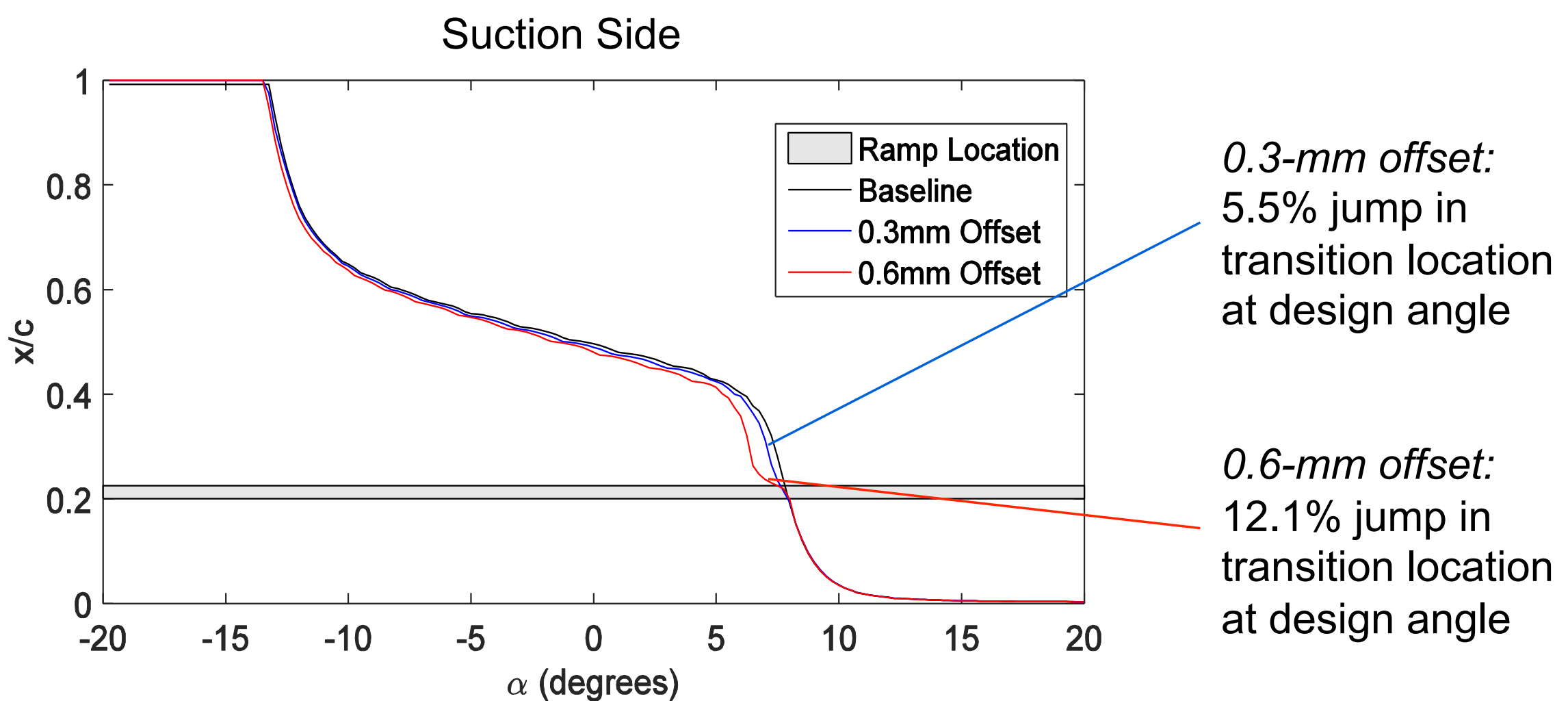

Boundary layer predictions from XFOIL support premature transition hypothesis 


\section{Fabric sections: literature}

Micro-aerial vehicle wings:

Low-aspect ratio, low Reynolds number, single-membrane wings

Increased camber increases $C \downarrow l$ and $C \downarrow d$; overall effect of these changes producing a $C \downarrow l / C \downarrow d$ comparable to similar rigid wings

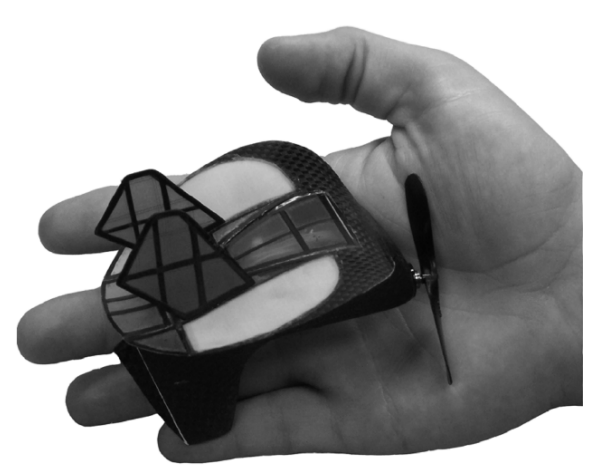

Stanford et al. (2007)

\section{Princeton sailwing:}

Higher aspect ratio, high Reynolds number, double-membrane wings

Lift curve nonlinear due to the changes in induced tension in the membrane with angle of attack

Predicted $C \downarrow l / C \downarrow d$ ratio for flexible wing similar to that of rigid wing and even exceeding in certain cases 


\section{Fabric section: configuration}
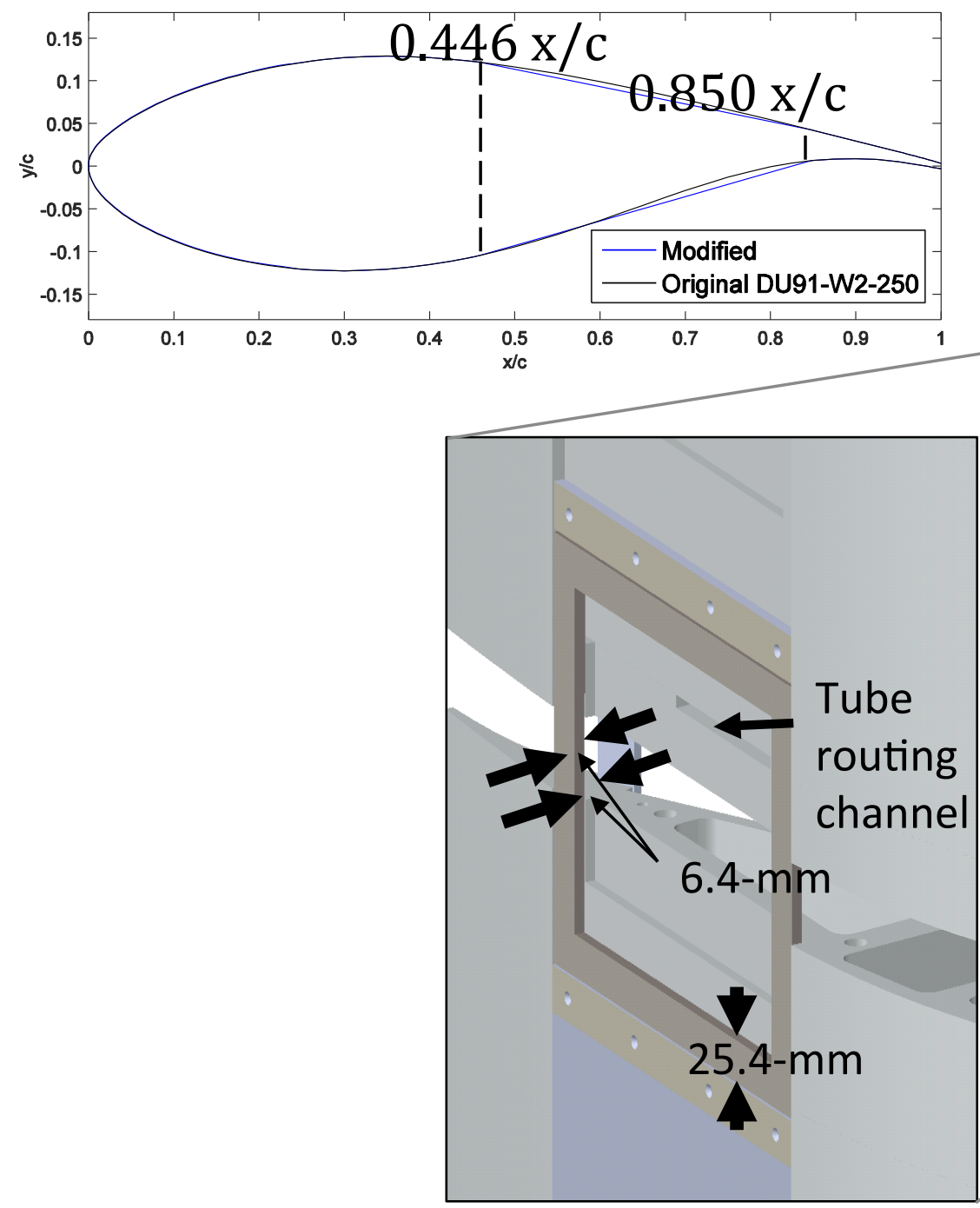

Middle panel

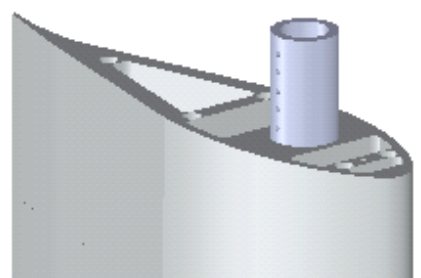




\section{Fabric section: test setup}
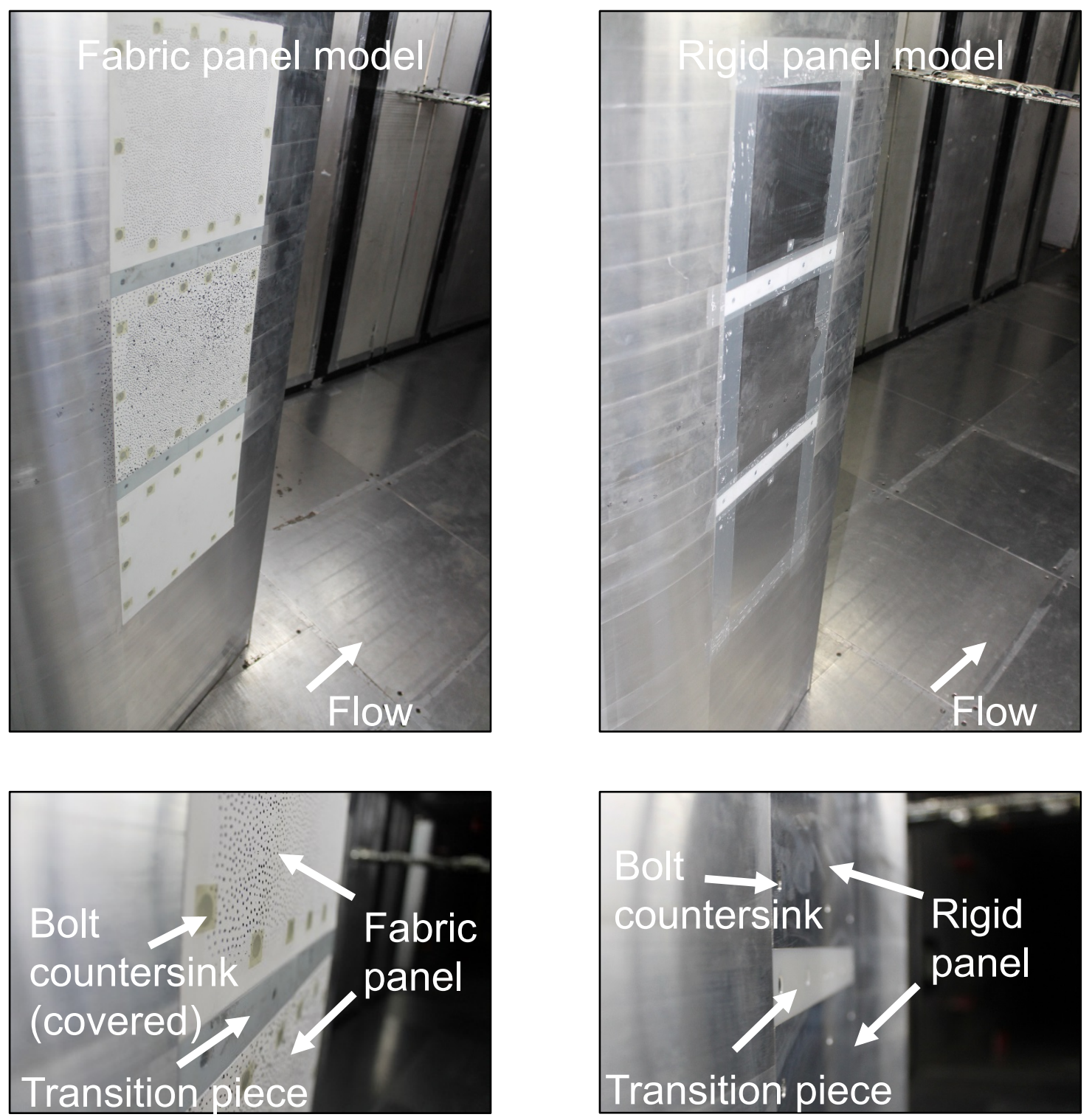

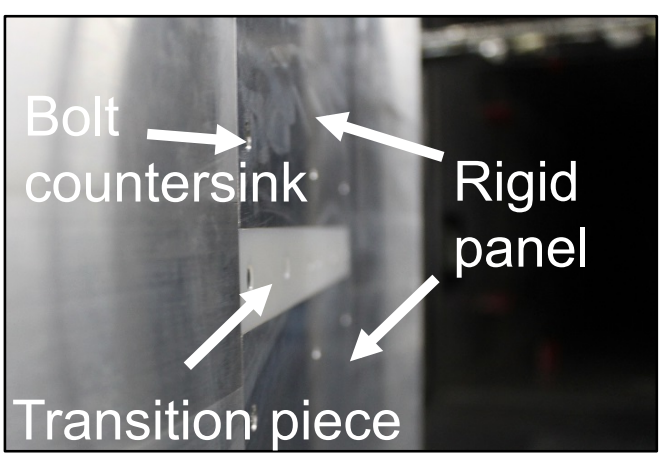

Model:

- Modified DU91-W2-250

- 0.8-m chord

- 34 aluminum laminates

- 64 pressure taps

- 6 removable panels (3 suction side, 3 pressure side)

- No endwall suction

Measurements:

- Mean surface pressure

- Wake profiles

- Fabric deflection (DIC)

Test Conditions:

- $\operatorname{Re}=2.5-3.0 \times 10^{6}$

- $\mathrm{Ma}=0.15-0.19$ 


\section{Fabric sections: results}

Modified (rigid) profile

Modified (fabric) profile

Modified (rigid) profile, XFOIL

Modified (fabric) profile, XFOIL: $z=0 \mathrm{~cm}$

Modified (fabric) profile, XFOIL: $z=6.5 \mathrm{~cm}$
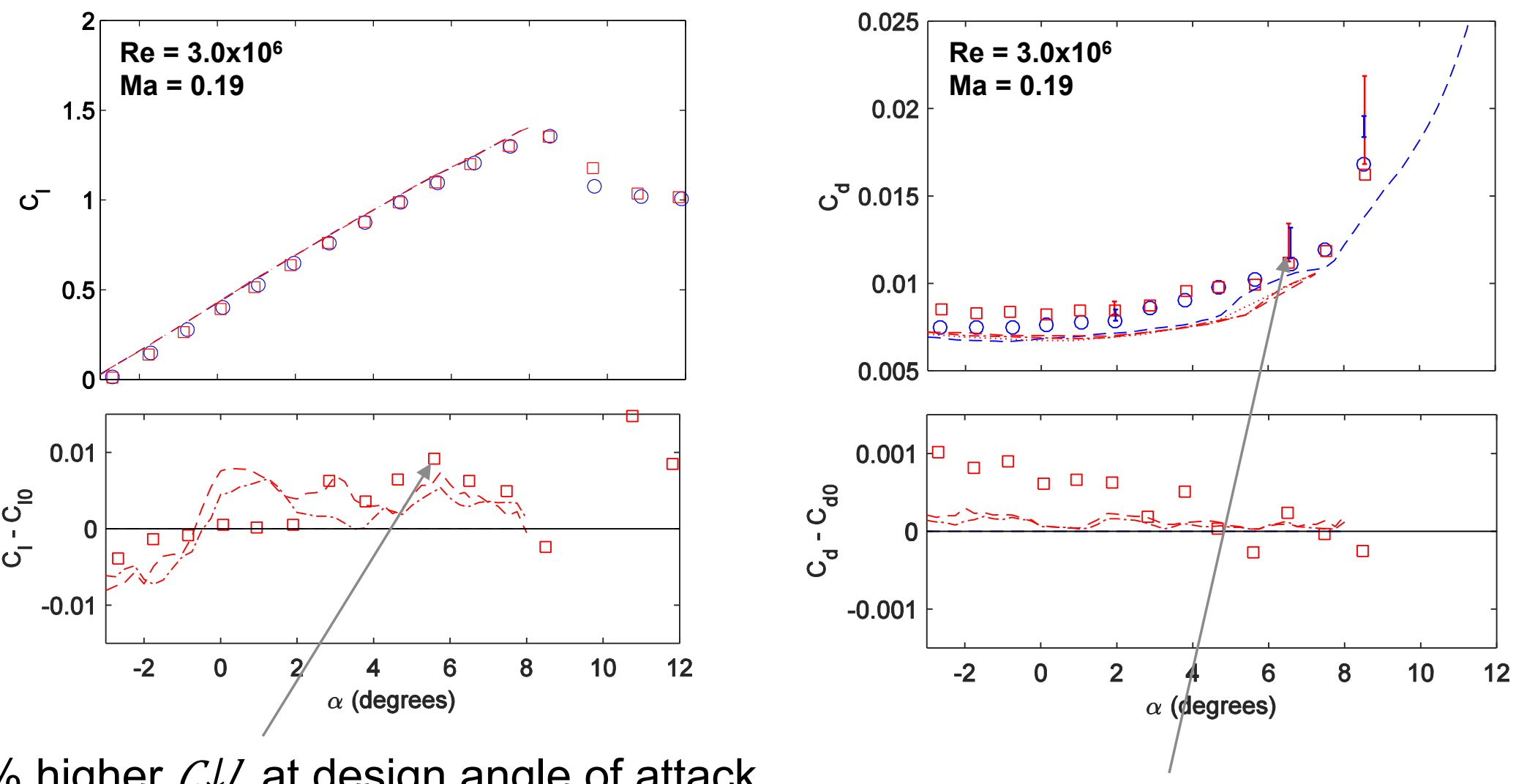

$0.8 \%$ higher $C \downarrow l$ at design angle of attack

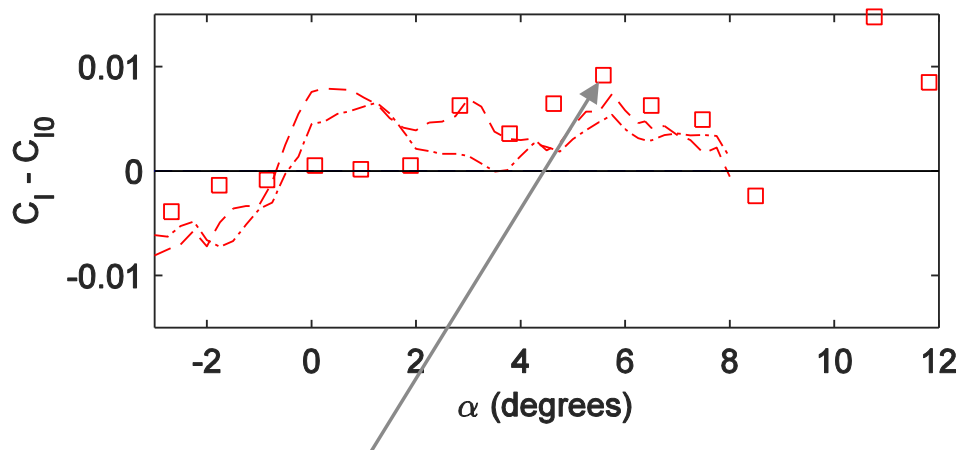

$19 \%$ variation in spanwise drag 


\section{Fabric sections: results}

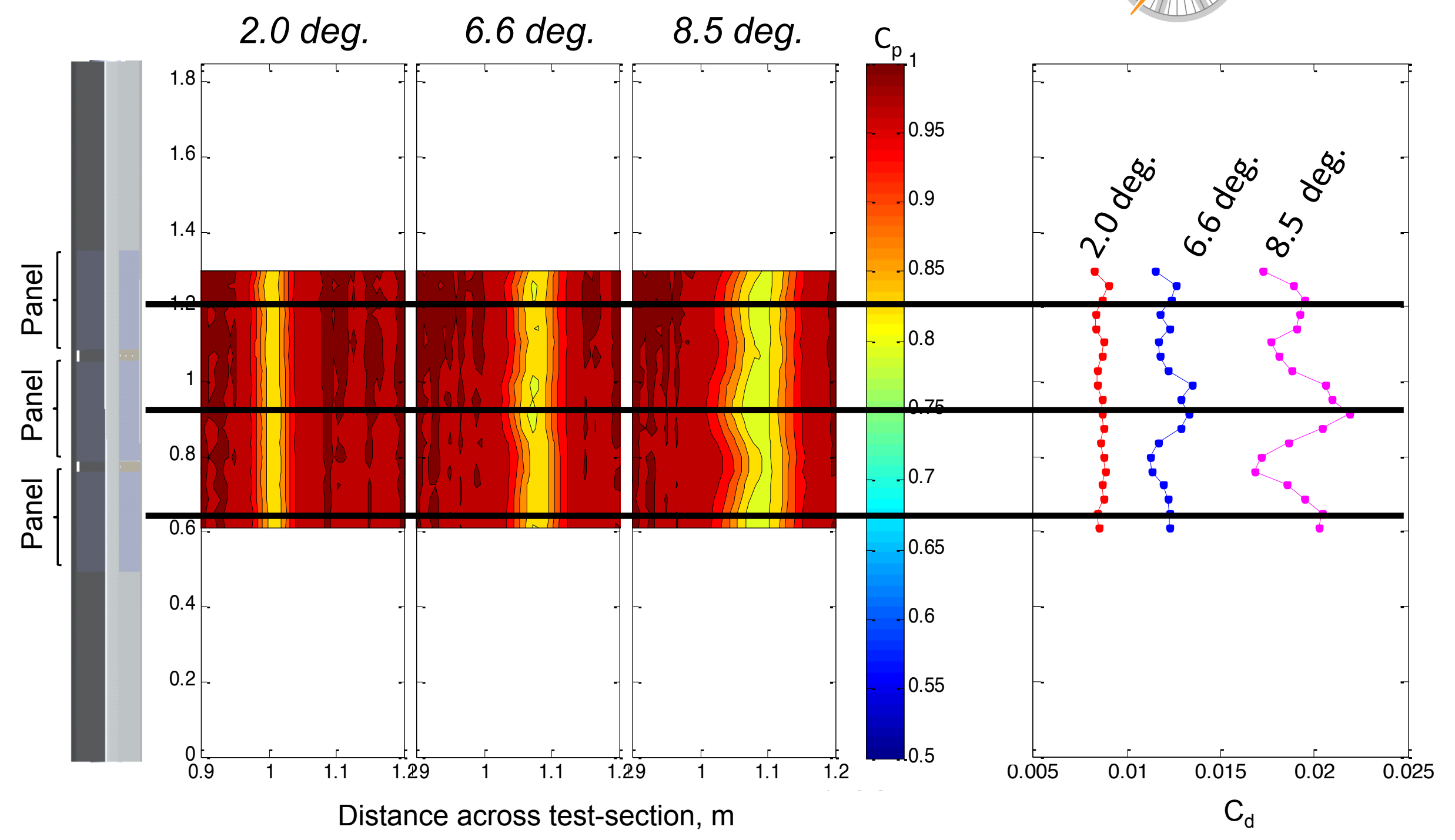

Fabric flexibility produces spanwise drag variation 


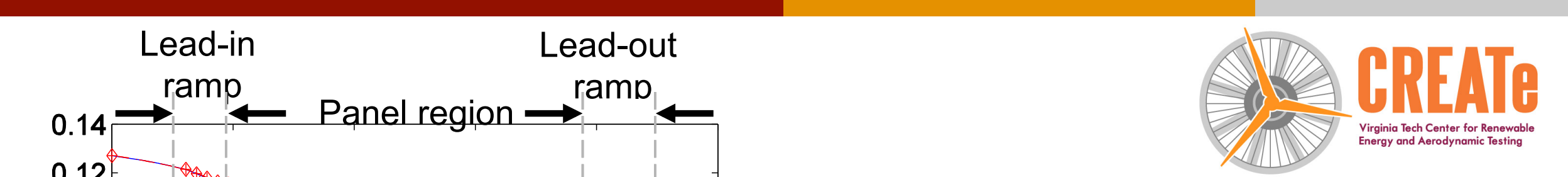

\section{Fabric sections: results}

\section{Suction side deflection}

Original profile has similar

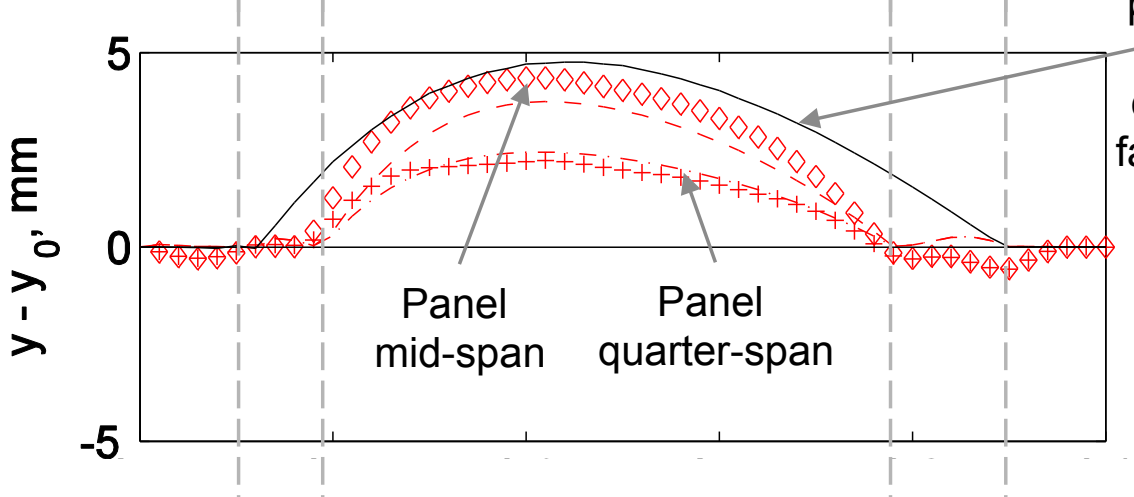
camber to fabric profile
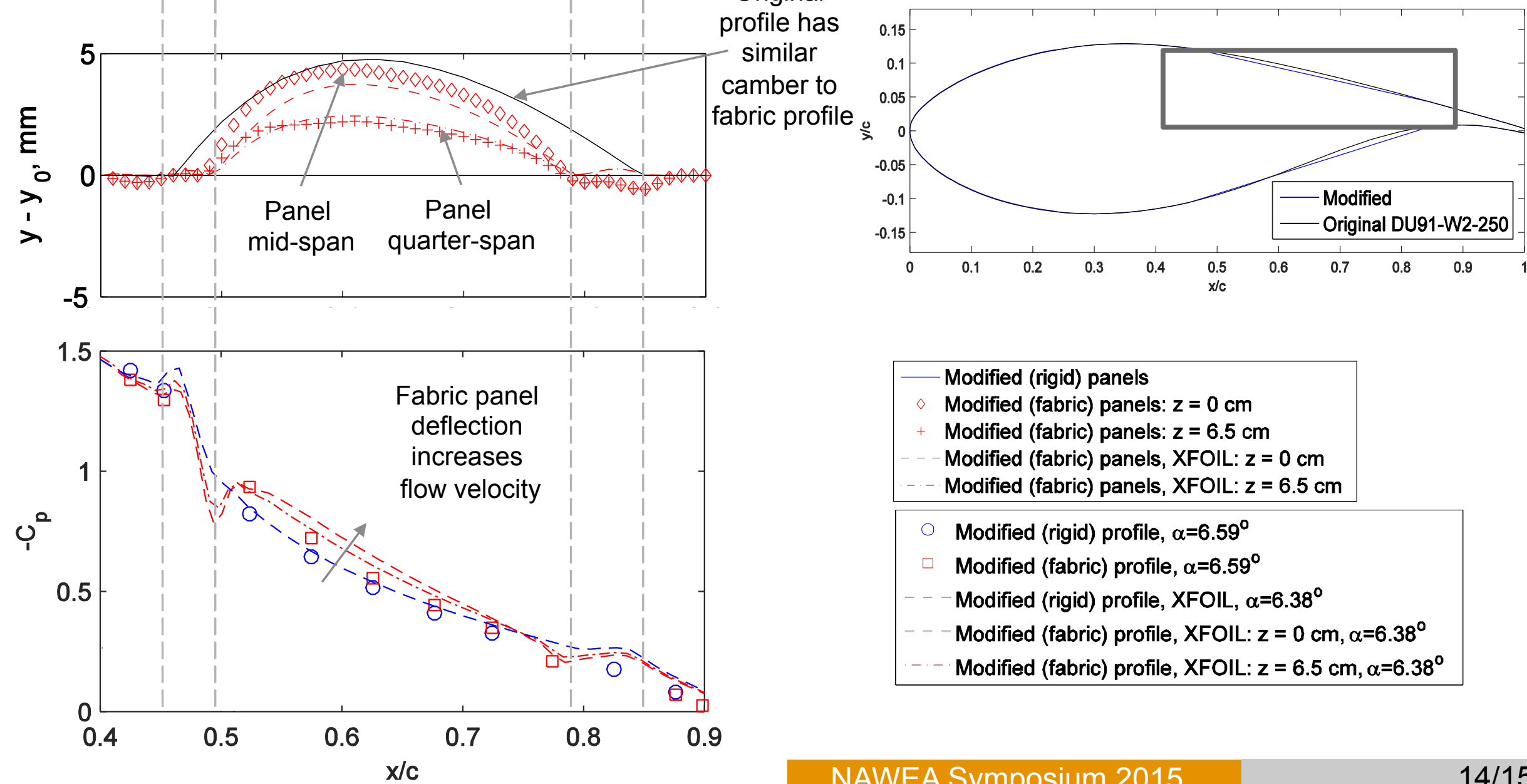

Modified (rigid) panels

Modified (fabric) panels: $z=0 \mathrm{~cm}$

Modified (fabric) panels: $z=6.5 \mathrm{~cm}$

Modified (fabric) panels, XFOIL: $z=0 \mathrm{~cm}$

Modified (fabric) panels, XFOIL: $z=6.5 \mathrm{~cm}$

Modified (rigid) profile, $\alpha=6.59^{\circ}$

Modified (fabric) profile, $\alpha=6.59^{\circ}$

Modified (rigid) profile, XFOIL, $\alpha=6.38^{\circ}$

Modified (fabric) profile, XFOIL: $z=0 \mathrm{~cm}, \alpha=6.38^{\circ}$

Modified (fabric) profile, XFOIL: $z=6.5 \mathrm{~cm}, \alpha=6.38^{\circ}$ 


\section{Summary/Conclusions}

\section{Misaligned model:}

-Small variation $(<1 \%)$ in linear region lift due to effective camber increase

-Premature transition responsible for increased drag and $(C \downarrow l / C \downarrow d) \downarrow$ max decrease of $\sim 5 \%$ for a typical misalignment and $\sim 17 \%$ for severe misalignment

\section{Fabric model:}

- $0.8 \%$ increase in $C \downarrow l$ for fabric versus baseline model at $(C \downarrow l / C \downarrow d) \downarrow$ max and above due to camber increase

- Large spanwise variation in drag due to fabric deflection requires detailed spanwise measurement

-Deflected fabric shape is not far from baseline shape, at least on suction side

-Fabric models afford opportunity for tuning performance with fabric properties

\section{Thank you!}




\section{References}

${ }^{1}$ Dutton, A., Kildegaard, C., Dobbe, T., Bensoussan, R., Kensche, C., Hahn, F., van Delft, D., and de Winkel, G., "Design, structural design, structural testing, and cost effectiveness of sectional wind turbine blades. (Publishable Final Report)," Tech. rep., The European Commission, 2000.

${ }^{2}$ Vionis, P., Lekou, D., Gonzalez, F., Mieres, J., Kossivas, T., Soria, E., Gutierrez, E., Galiotis, C., Philippidis, T., Voutsinas, S., and Hofmann, D., "Development of a MW scale wind turbine for high wind complex terrain sites; the MEGAWIND project." Tech. rep., 2006 European Wind Energy Conference, 2006.

${ }^{3}$ Saenz, E., Nuin, I., Montejo, R., and Sanz, J., "Development and validation of a new joint system for sectional blades," Wind Energy, Vol. 18, No. 3, 2014, pp. 419428.

${ }^{6}$ Braslow, A. L., "Review of the effect of distributed surface roughness on boundary-layer transition," Tech. rep., DTIC Document, 1960.

${ }^{7}$ Fage, A., "The smallest size of a spanwise surface corrugation which affects boundary-layer transition on an aerofoil," HM Stationery Office, 1943.

${ }^{8}$ Smith, A., "The smallest height of roughness capable of affecting boundary-layer transition," Journal of the Aerospace Sciences, Vol. 26, No. 4, 1959, pp. 229-245.

${ }^{10}$ Drake, A., Bender, A. M., Korntheuer, A., Westphal, R. V., McKeon, B. J., Gerashchenko, S., Rohe, W., and Dale, G., "Step Excrescence Effects for Manufacturing Tolerances on Laminar Flow Wings," AIAA Paper, Vol. 375, 2010.

${ }^{18}$ Stanford, B., Sytsma, M., Albertani, R., Viieru, D., Shyy, W., and Ifju, P., "Static aeroelastic model validation of membrane micro air vehicle wings," AIAA journal, Vol. 45, No. 12, 2007, pp. 2828-2837.

${ }^{19}$ Ormiston, R. A., "Theoretical and experimental aerodynamics of the sailwing," Journal of Aircraft, Vol. 8, No. 2, 1971, pp. $77-84$. 


\section{Brown et al.}




\section{DU00}




\section{Misaligned sections: literature}

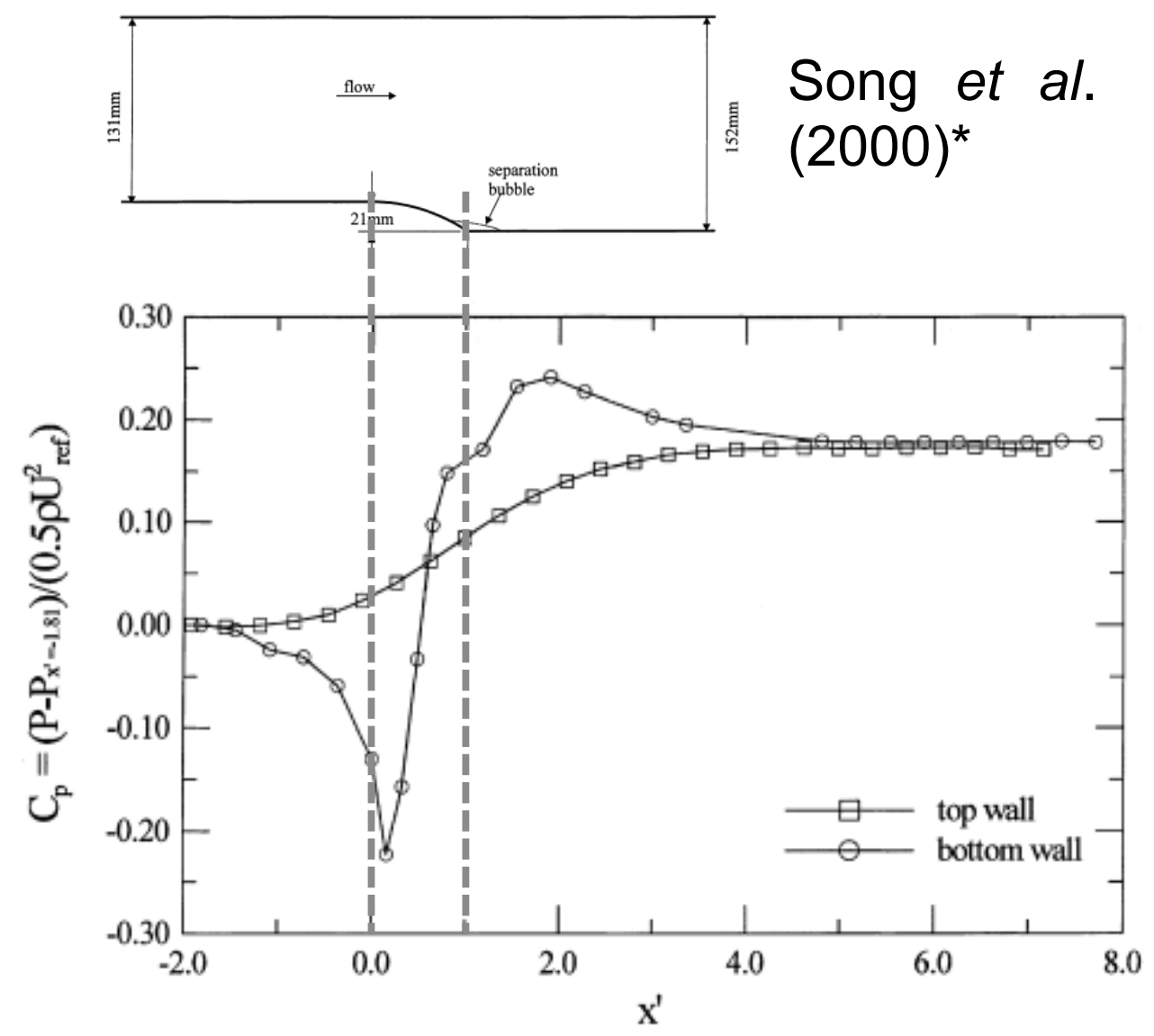

*Song et al. (2000) - Experimental study of a separating, reattaching, and redeveloping flow over a smoothly contoured ramp 


\section{Misaligned sections: setup}

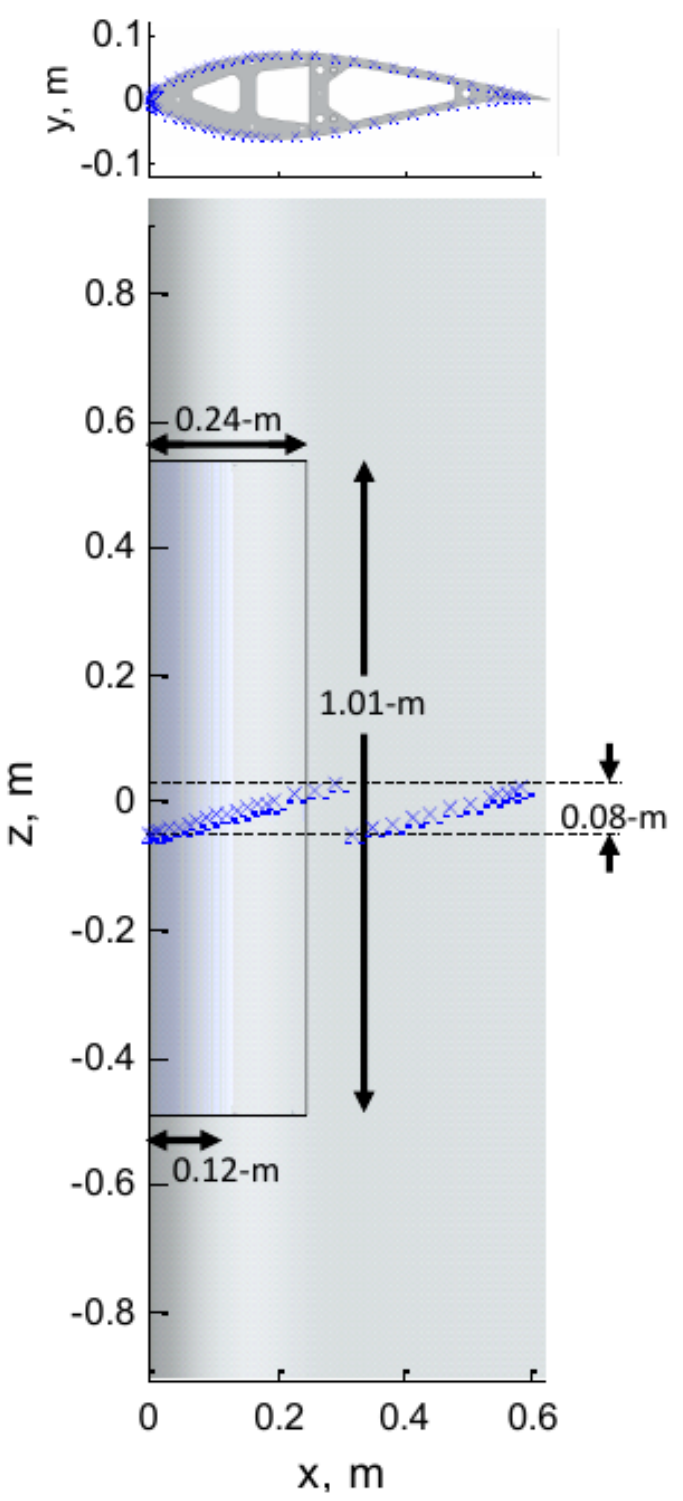




\section{Effective Angle of Attack Increase}

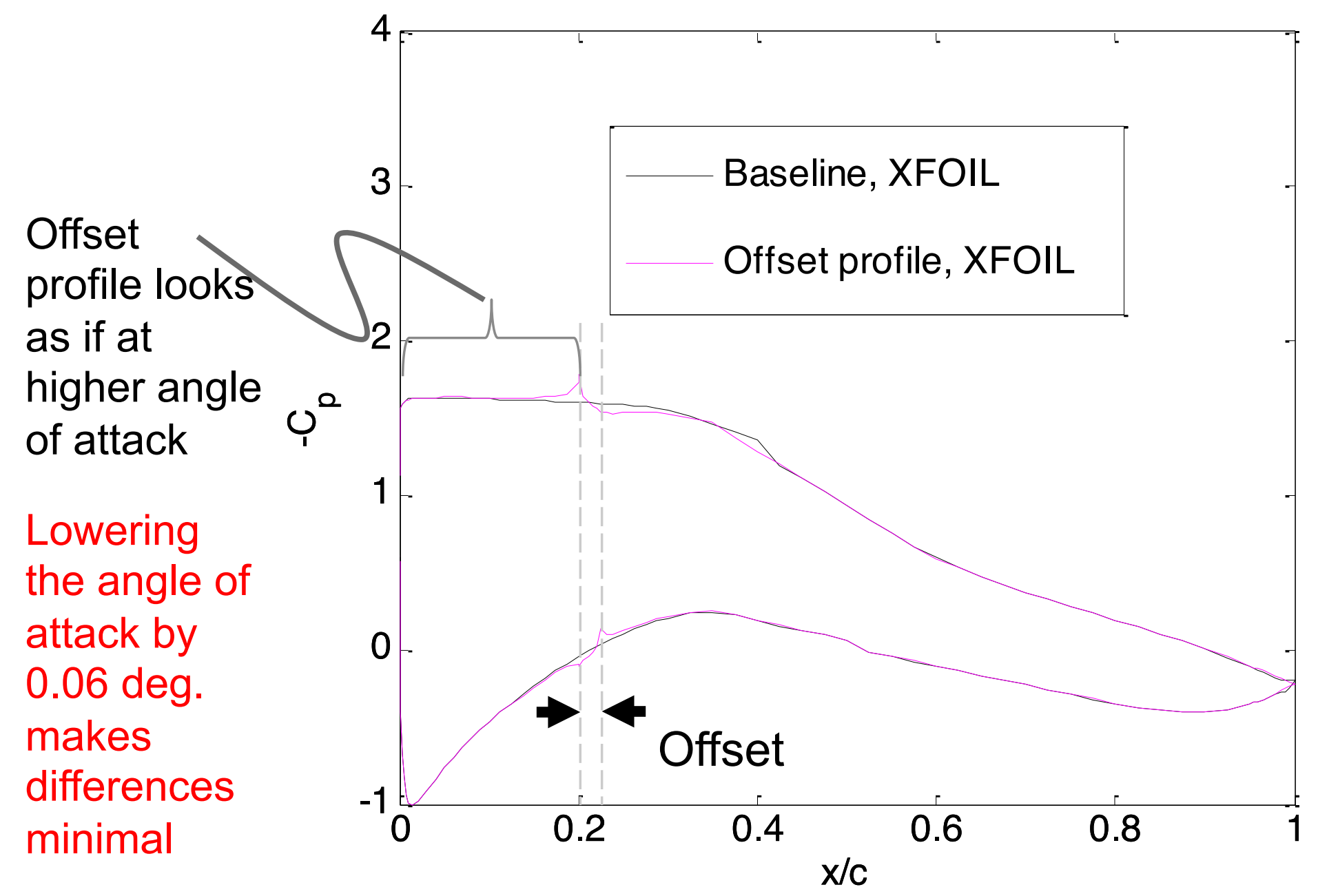

Offset effect predominantly at nose 


\section{Misaligned sections: results}

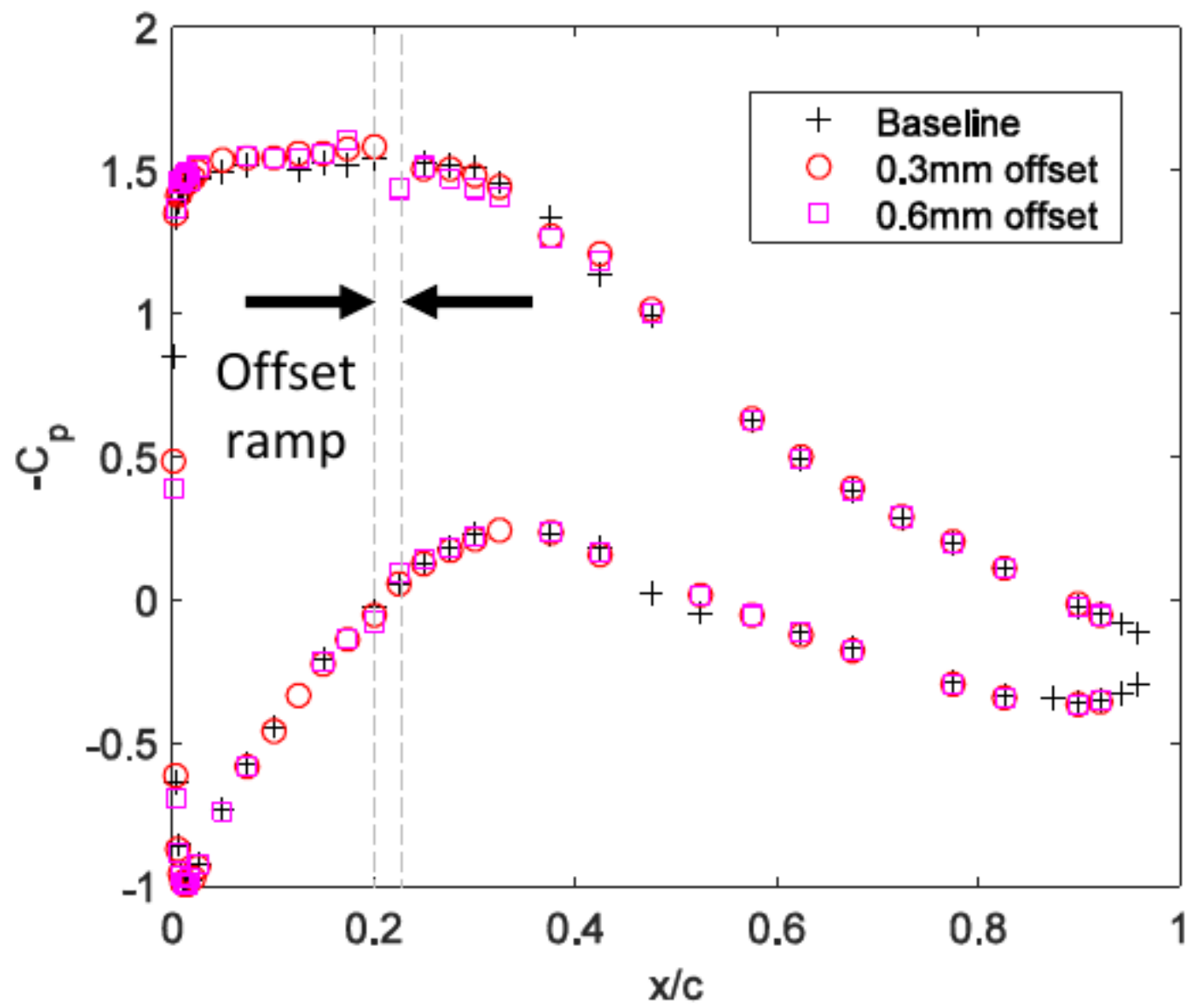




\section{Misaligned sections: results}

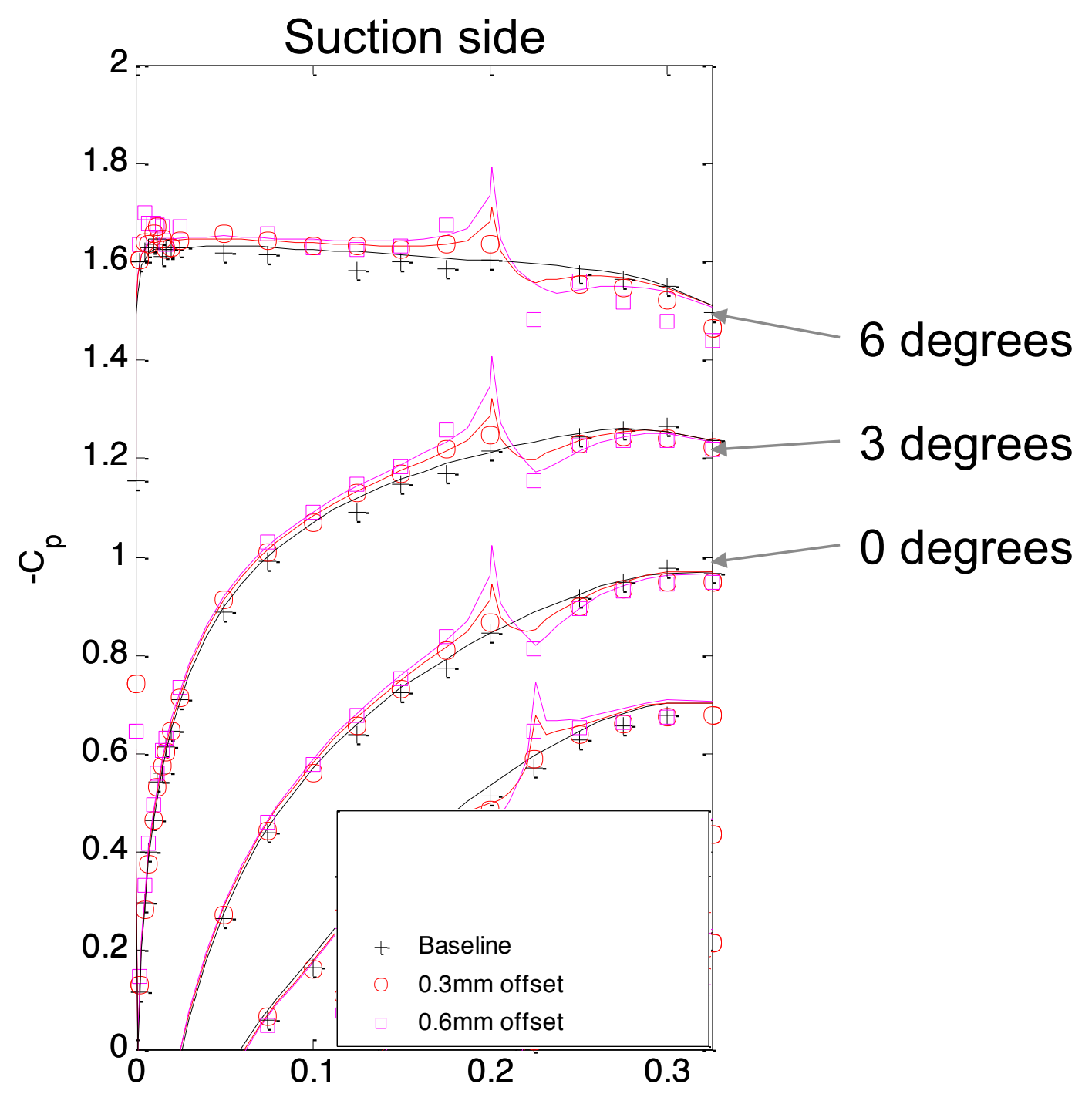




\section{Misaligned sections: results}

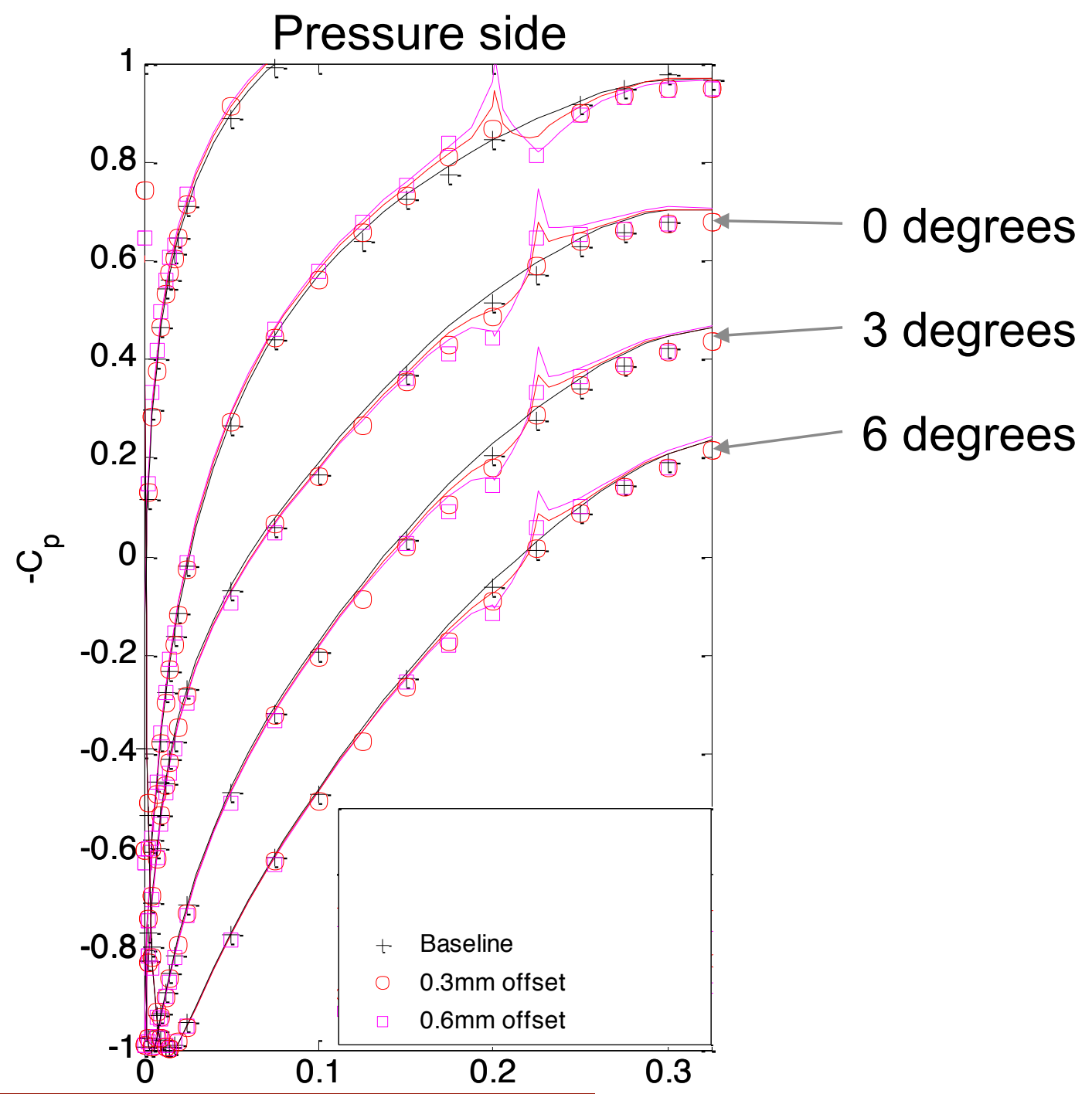




\section{Boundary layer comparison at 6}

deg.

(from XFOIL)

0.009

0.008

0.007

Baseline

$0.3 \mathrm{~mm}$ offset

$0.6 \mathrm{~mm}$ offset

0.006

* 0.005

0.004

0.003

0.002

0.001

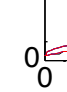

0.1

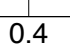

0.5

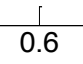

0.7

0.8

0.9 


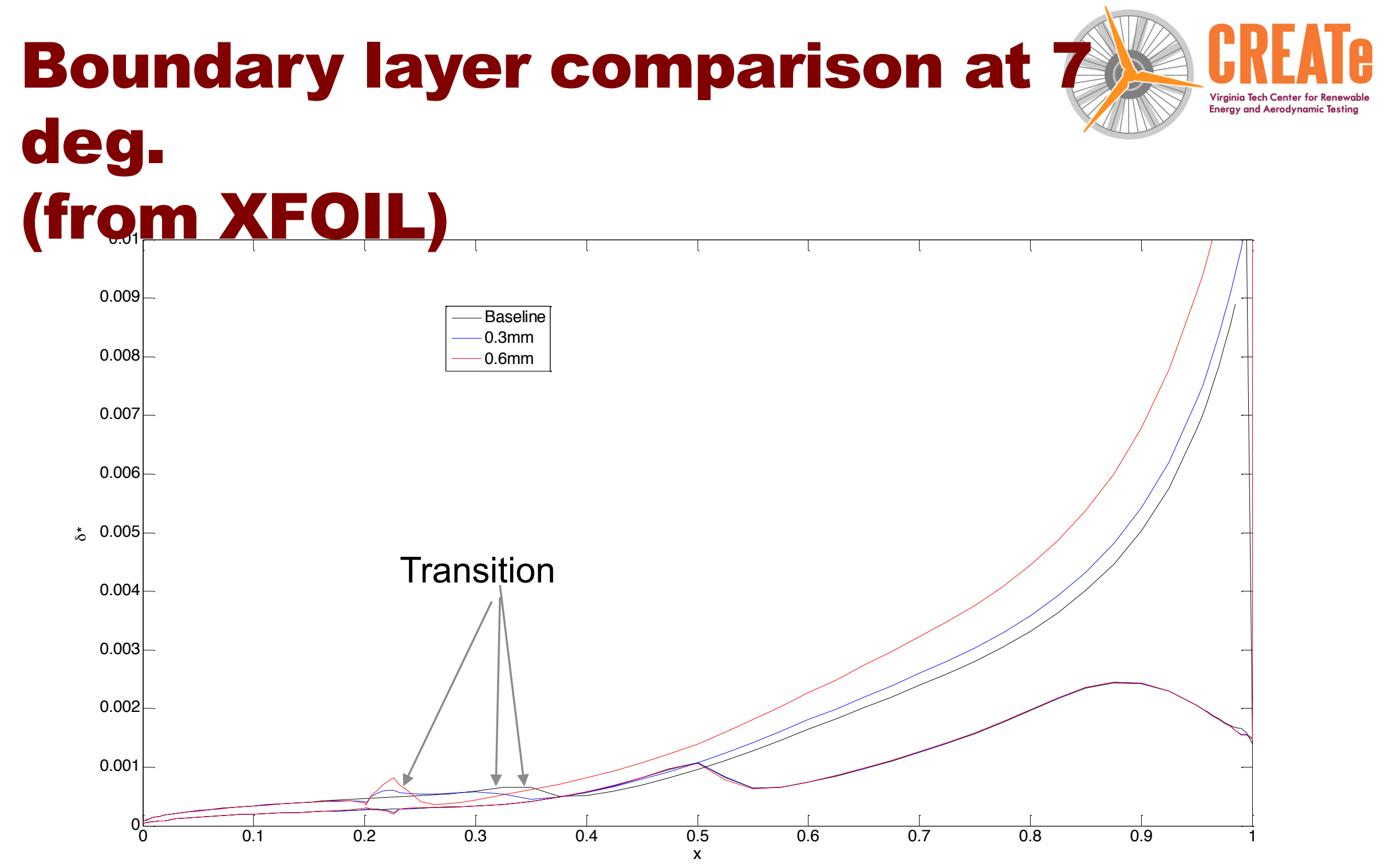




\section{Boundary layer comparison at 8}

deg.

(from XFOIL)

0.009

0.008

0.007

- Baseline

0.006

$0.3 \mathrm{~mm}$

0.006

* 0.005

0.004

0.003

0.002

0.001

0

0.1

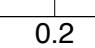

Transition 


\section{Misaligned sections: results}
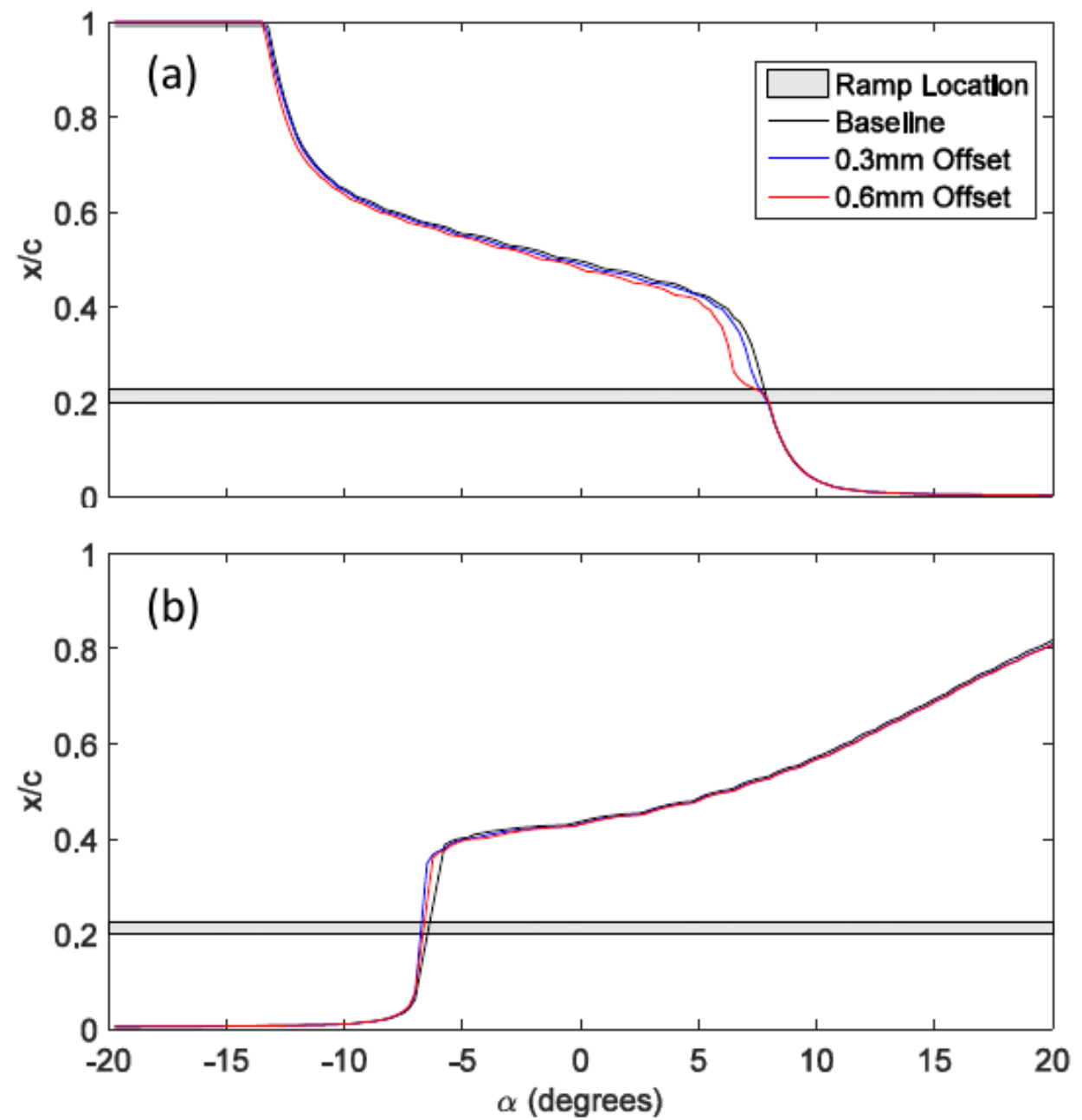


\section{DU91}




\section{Fabric sections: literature}

Smith and Shyy ${ }^{18}$ note that the static aeroelastic problem for a single membrane in two-dimensions can be fully described by five non-dimensional variables: Reynolds number, angle of attack, excess ratio (equal to zero for a taut membrane), non-dimensionalized elastic modulus

$$
\Pi_{1}=\left(E h / q_{\infty} c\right)^{1 / 3}
$$

and non-dimensionalized pres-stress

$$
\Pi_{2}=\left(S h / q_{\infty} c\right)^{1 / 3}
$$

where $E$ is the elastic modulus of the membrane, $S$ is the pre-stress of the membrane, $h$ is the thickness of the membrane, $q_{\infty}$ is the dynamic pressure of the freestream flow, and $c$ is the chord of the wing. Depending on whether the membrane tension is dominated by pre-stress or elastic stress, the relative importance of $\Pi_{1}$ versus $\Pi_{2}$ in the out-of-plane equilibrium are interchanged. 


\section{Fabric sections: configuration}

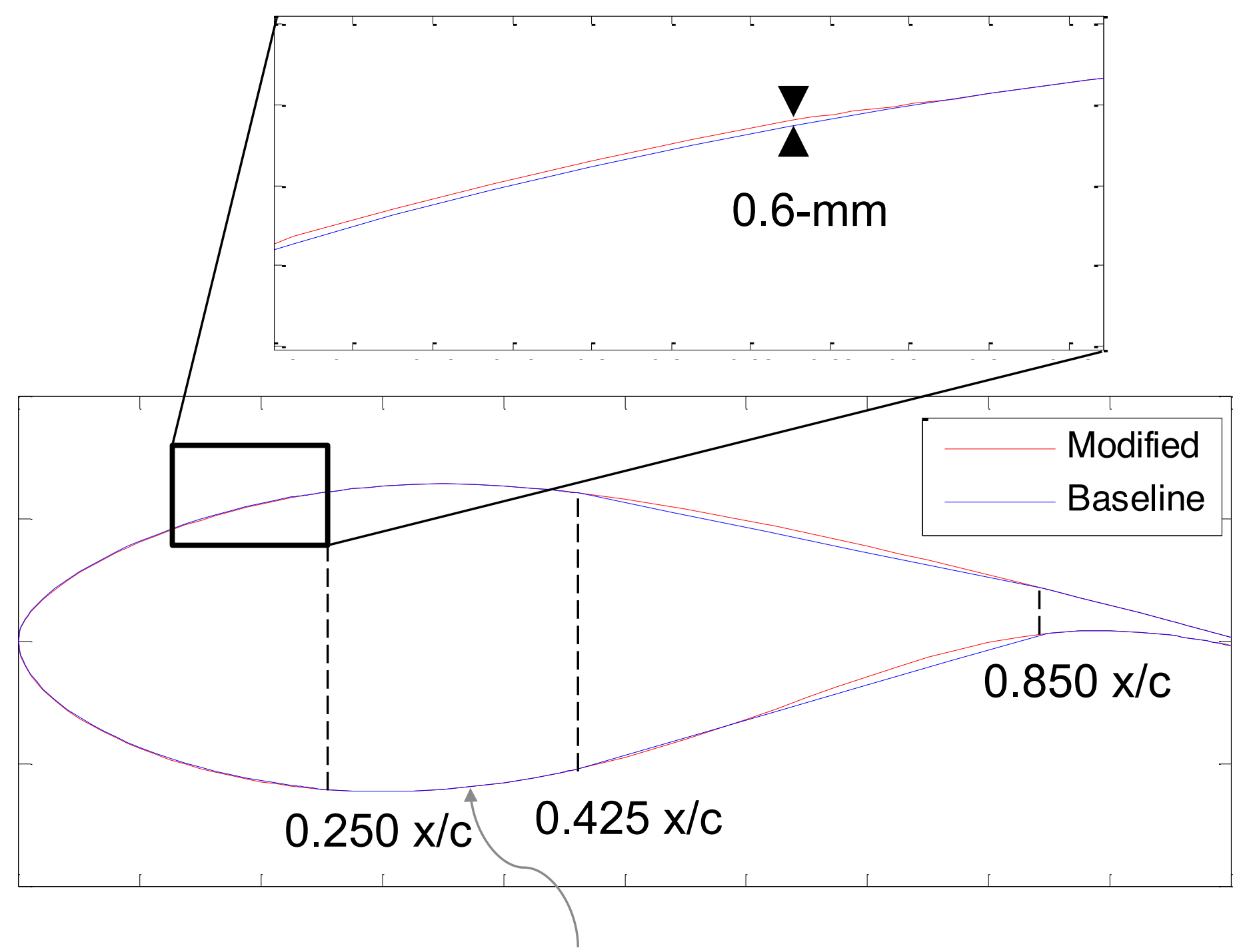

DU91-W2-250 profile 


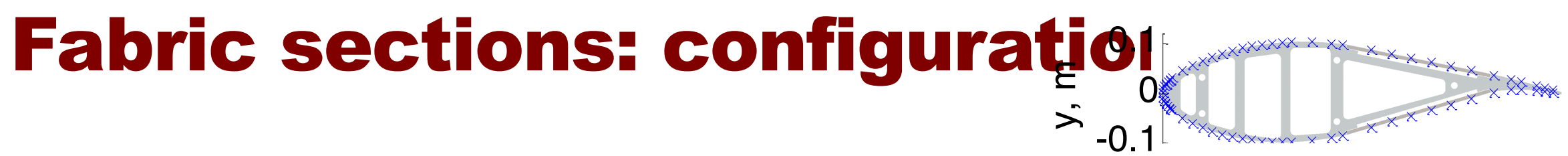
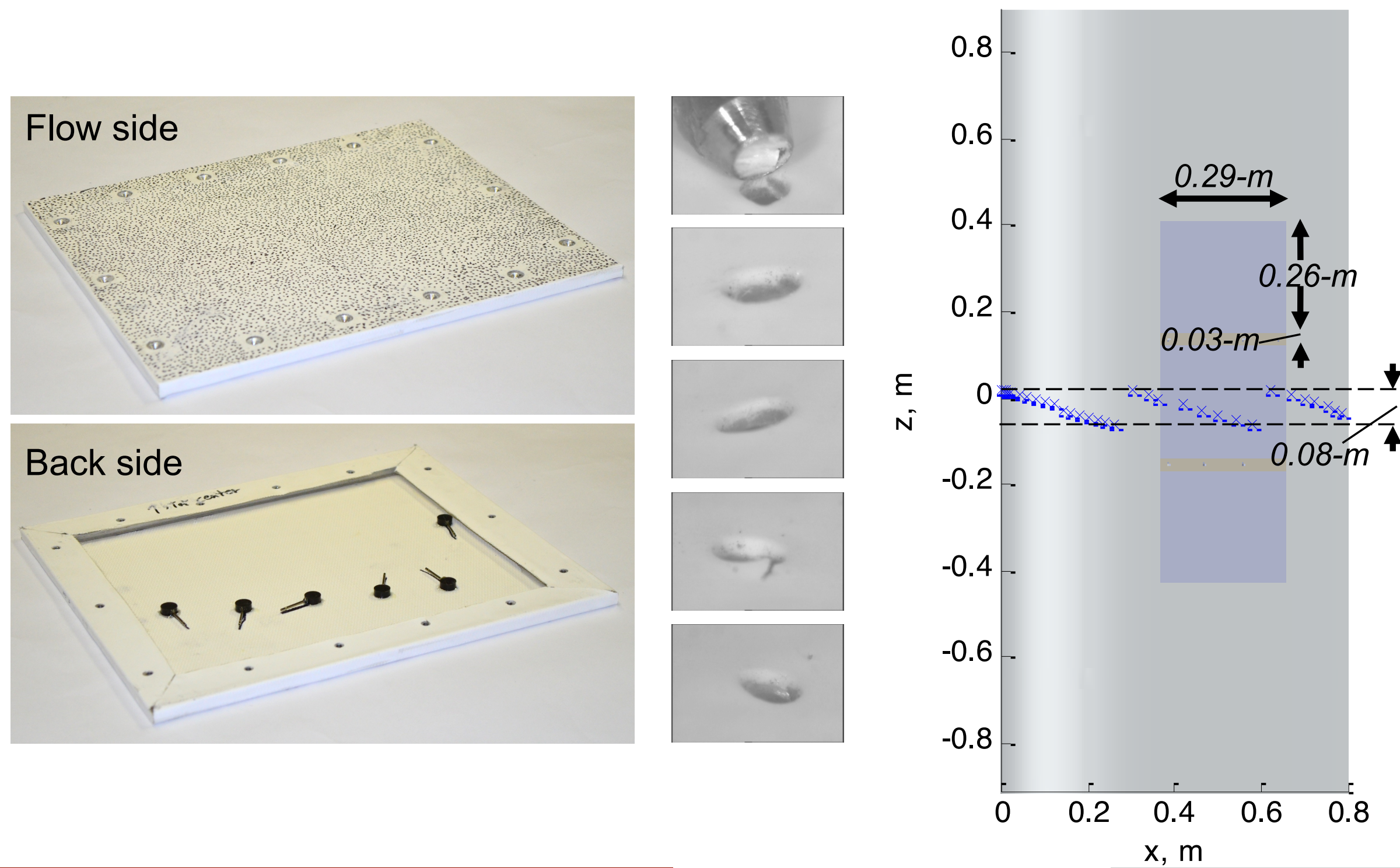


\section{Fabric sections: setup}
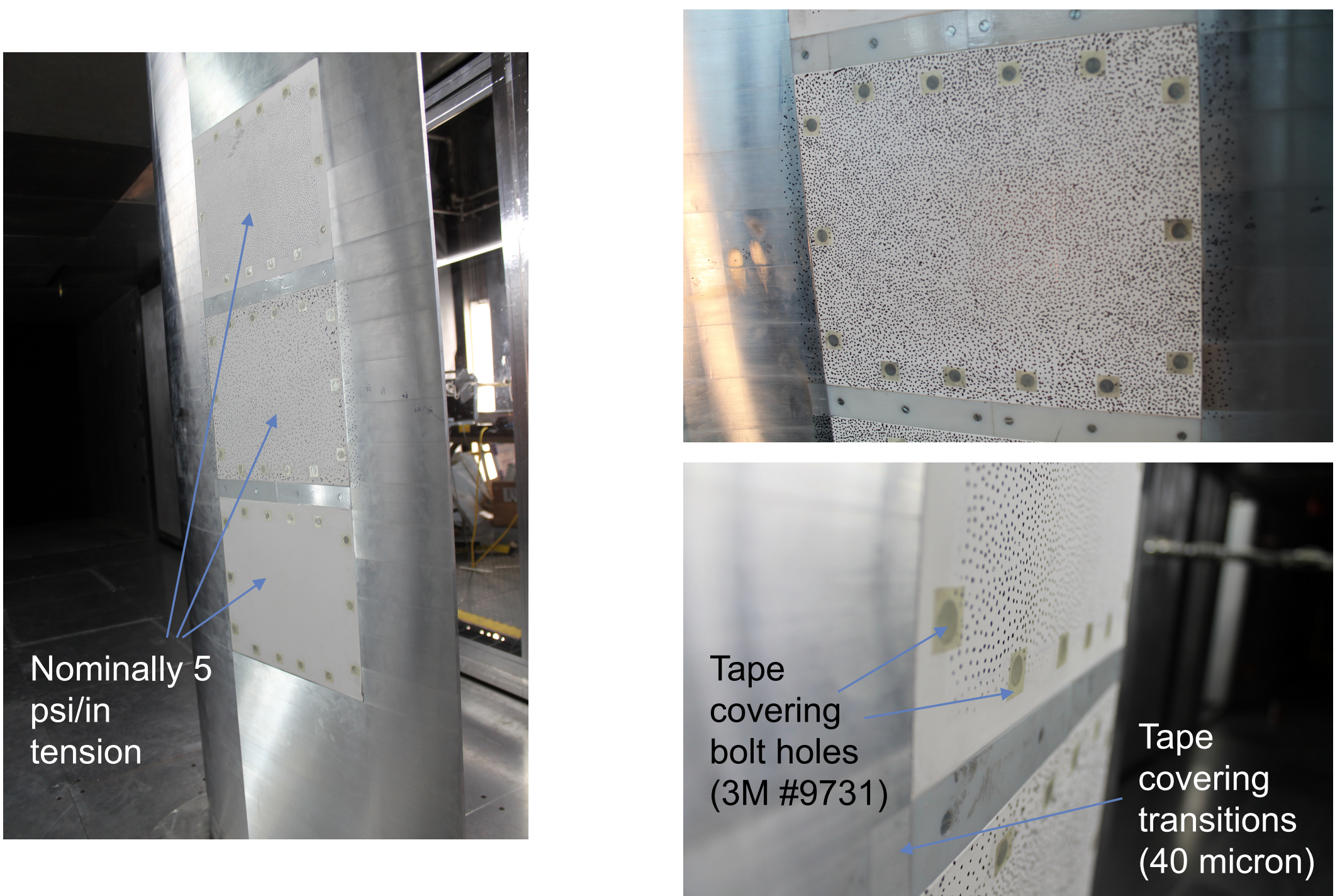

No taping on leading or trailing edges of fabric panels 


\section{Fabric sections: setup}
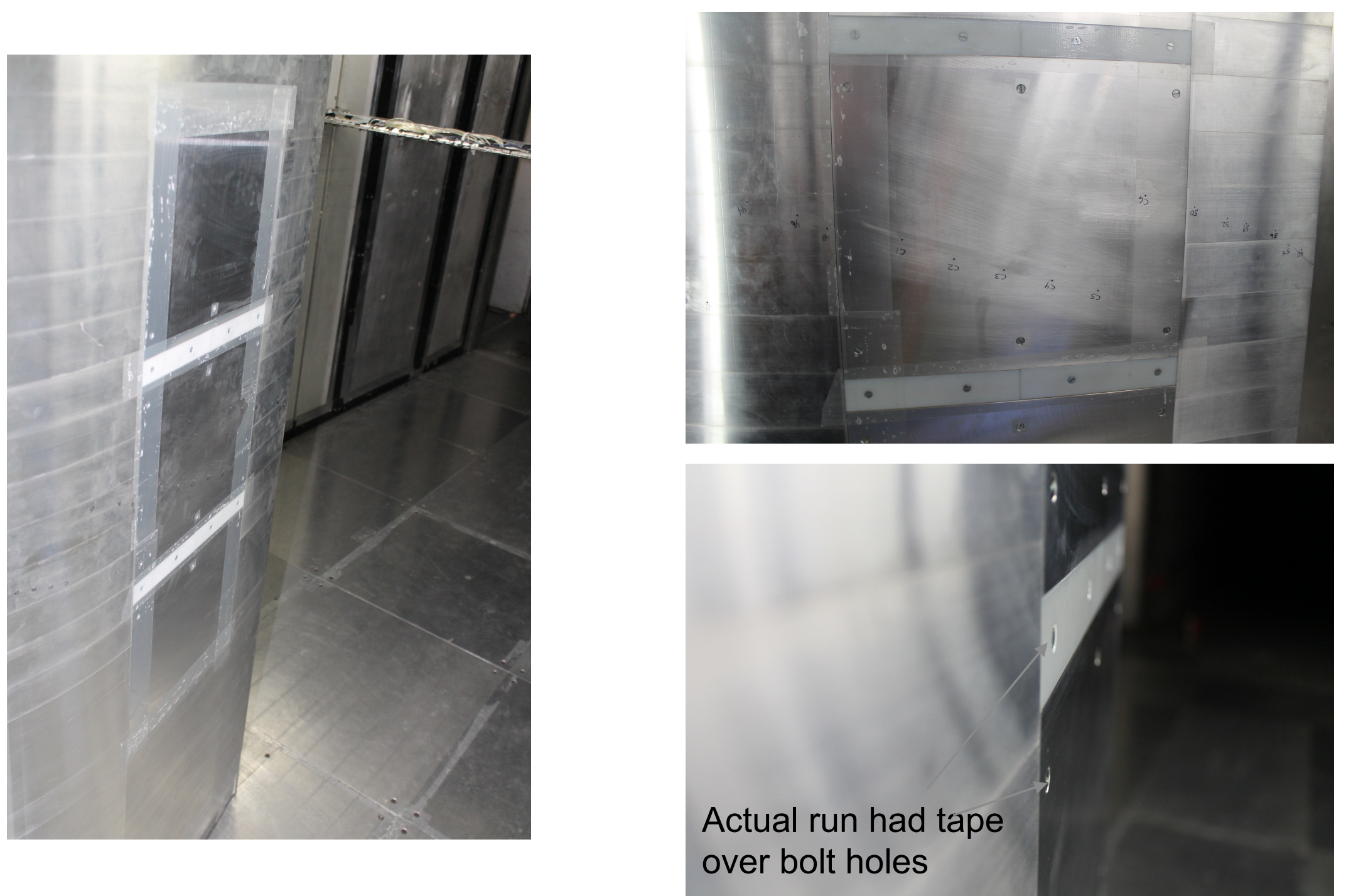

All panel joints covered with 40 micron tape 


\section{Fabric sections: setup}
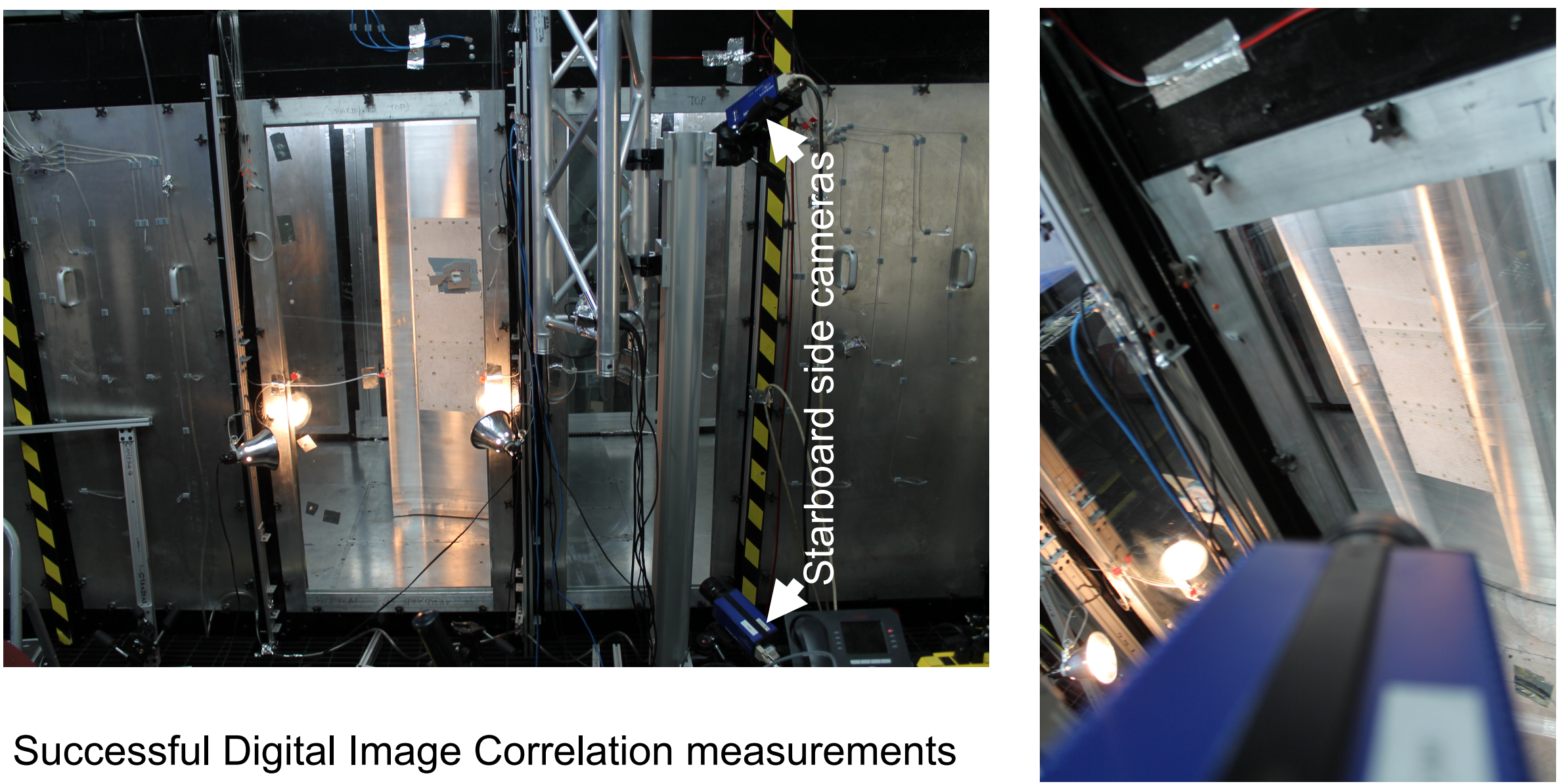

Successful Digital Image Correlation measurements 


\section{Design of DIC System}

Experimental setup variables:

- $b$-baseline separation of cameras

- $\quad Z$ - depth to measurement space

- $f$ - lens' focal length

- $m=$ magnification

- $\quad N$ - f-stop number $(f /$ aperture)

- $\Delta d$-physical pixel spacing

- $h$-number of pixels in 1D

- $F O V$ (field-of-view) -extent of measurement space in $1 \mathrm{D}$

- DOF (depth-of-field) - out-ofplane distance in-focus

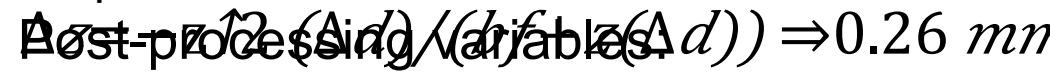

- $H$ - circle of confusion diameter

$D O F=-2 H N(m+1) / m \uparrow 2 \Rightarrow 0.42 m$
LaVision StrainMaster 2D/3D DIC system:

- 2 Imager Pro X 4M

- 4 MP cameras

- 52-mm lenses

- 2,048-by-2,048 resolution

- 7.4-by-7.4 $\mu \mathrm{m}$ pixel spacing

- $14 \mathrm{~Hz}$ sampling

- 14 bit digital output 


\section{Iteration on Design}

for Rotating Truss-Mounted Cameras

$f=h z(\Delta d) / F O V+h(\Delta d) \Rightarrow 24.03$

$\mathrm{mm}$

$\Delta z=-\mathrm{z} \uparrow 2(\Delta d) /(b f+z(\Delta d)) \Rightarrow 0.66 \mathrm{~mm}$

$D O F=-2 H N(m+1) / m \uparrow 2>10 \mathrm{~cm}$ (if aperture $<$ $6 \mathrm{~mm}$ )

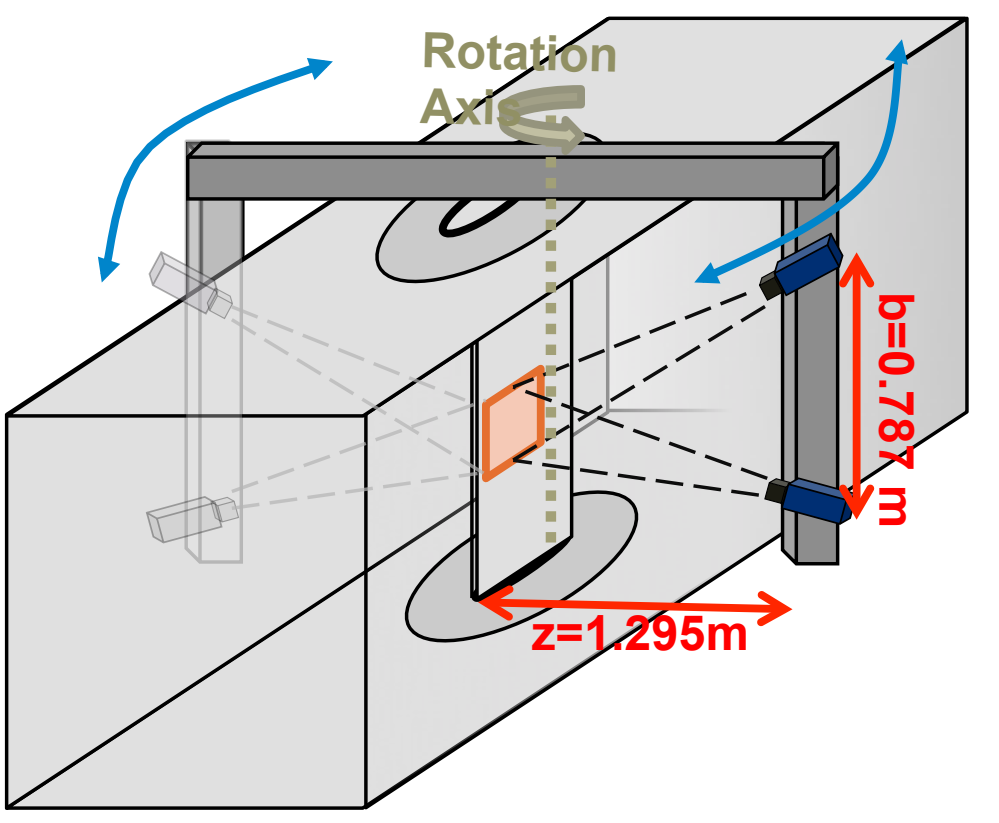




\section{Iteration on Design}

for Rotating Truss-Mounted Cameras

$f=h z(\Delta d) / F O V+h(\Delta d) \Rightarrow 24.03$

$m m$

$\Delta z=-\mathrm{z} \uparrow 2(\Delta d) /(b f+z(\Delta d)) \Rightarrow 0.66 \mathrm{~mm}$

$D O F=-2 H N(m+1) / m \uparrow 2>10 \mathrm{~cm}$ (if aperture $<$ $6 \mathrm{~mm}$ )

New Side Windows (1/2" thick laminated glass)

- Uncertainty due to glass refraction:

Sutton and McFadden (2000): water tunnel experiment, rotation of the cameras of 12 degrees relative to viewing window induced a strain error of $10,000 \mu$ strain or $0.01 \mathrm{~m} / \mathrm{m}$

- Structural integrity of glass:

Viewing angle requirement on dimensions: $1.42 \mathrm{~m}$ streamwise by $1.60 \mathrm{~m}$ spanwise (as measured by Tim Meyers) 


\section{Fabric sections: results $R e=3.5 \mathrm{M} \rightarrow 0(70 \mathrm{~m} / \mathrm{s} \rightarrow 0)$}

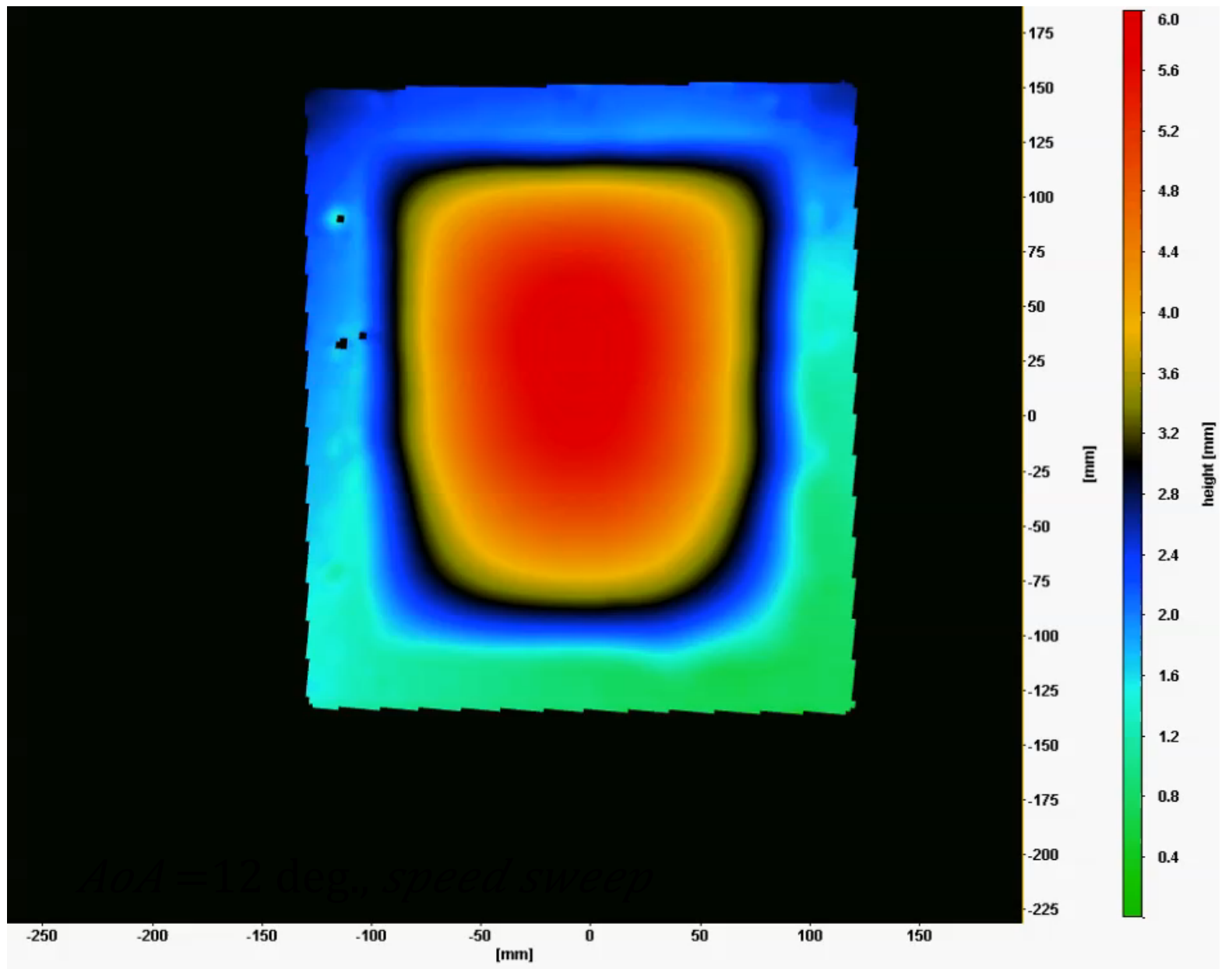




\section{Fabric sections: results}

Camber lines at 6 deg., $\operatorname{Re}=3 \mathrm{M}$

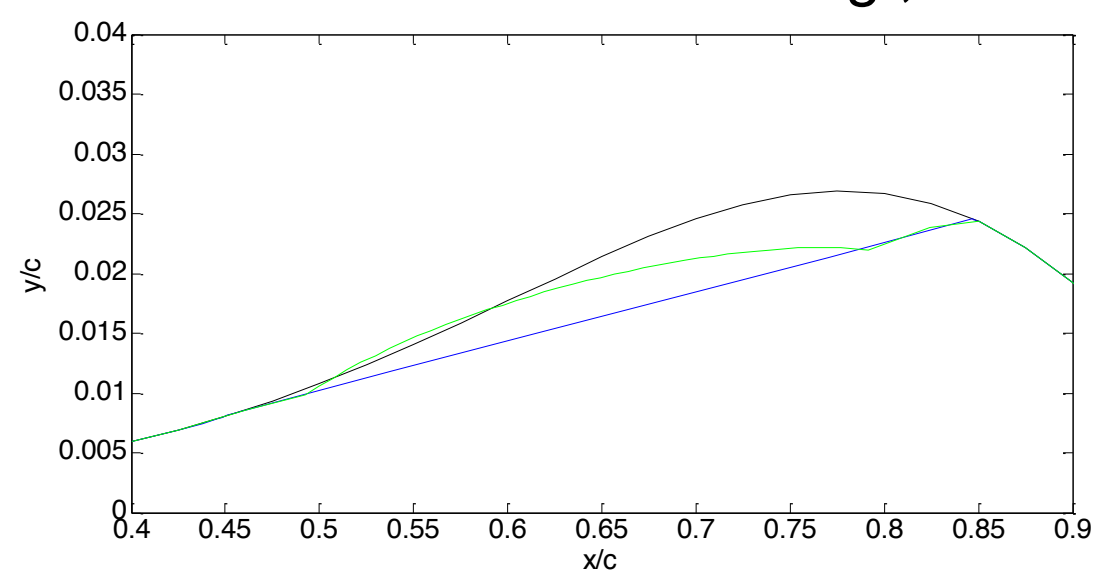

Baseline

Modified (rigid) profile

Modified (fabric) profile, predicted 


\section{Fabric sections: results}

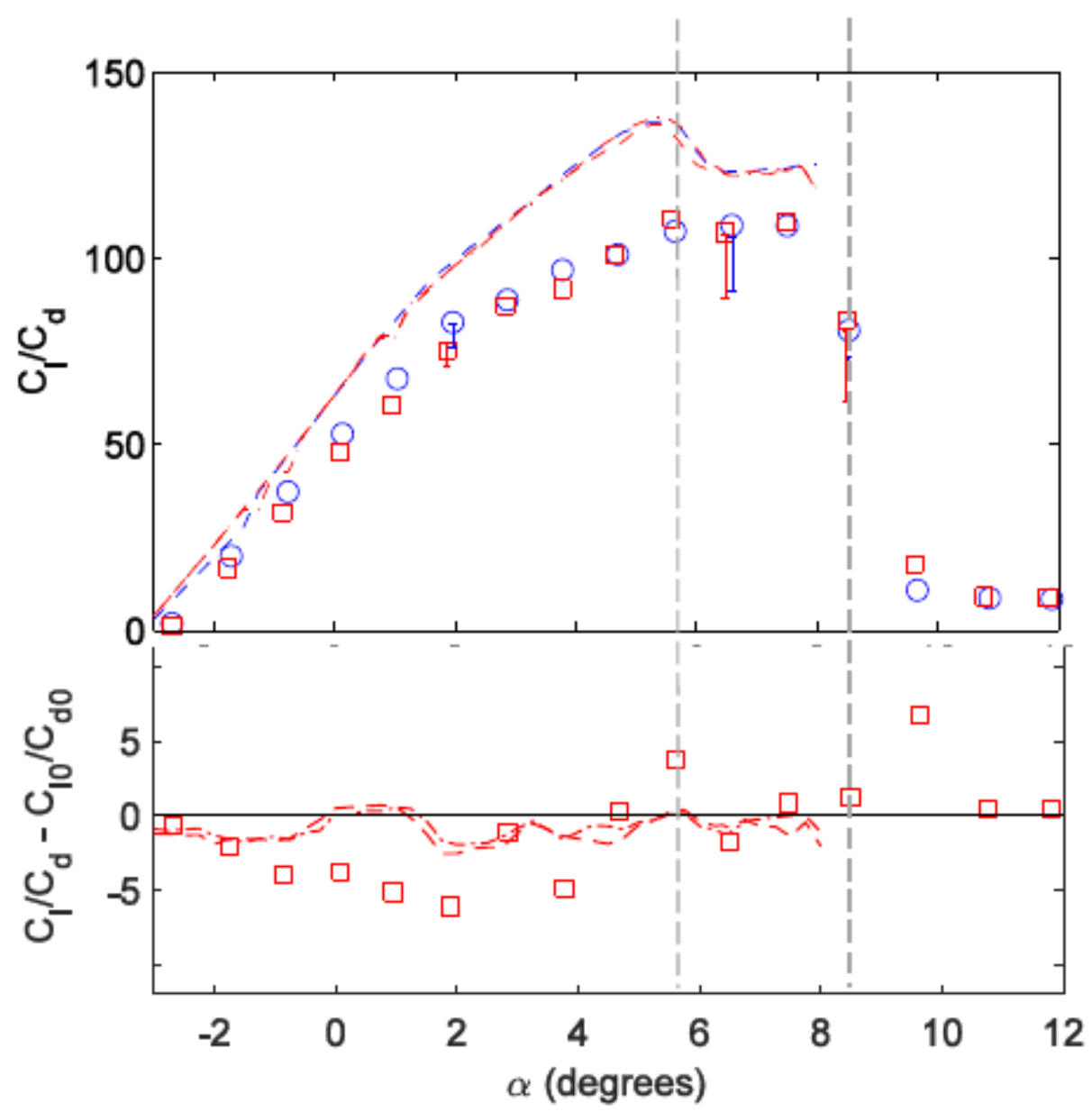




\section{Fabric sections: results}

(a) Suction Side

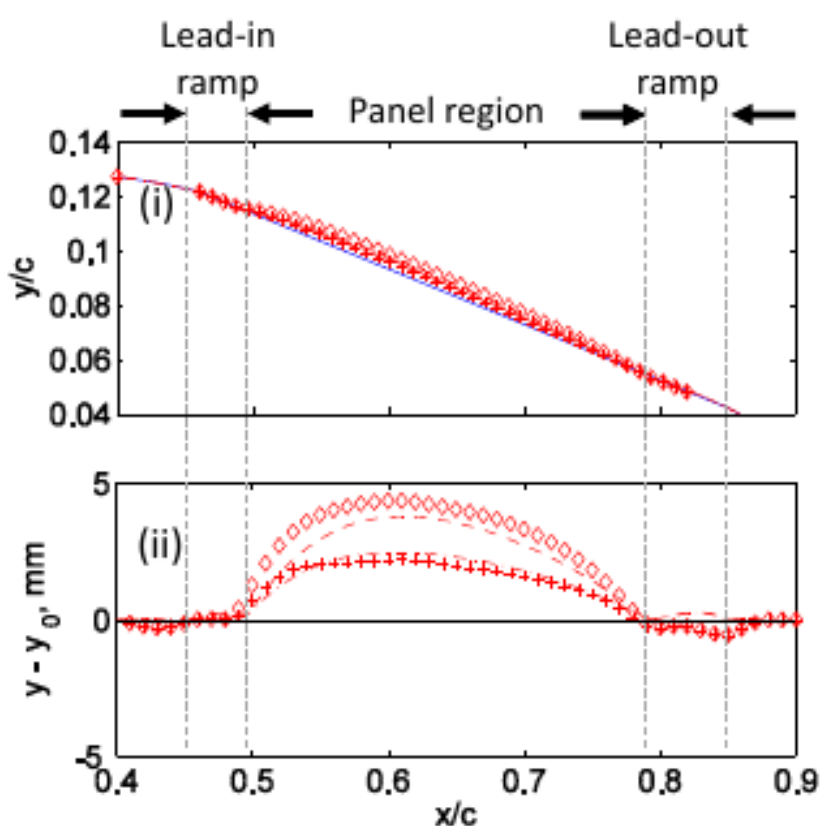

(b) Pressure Side

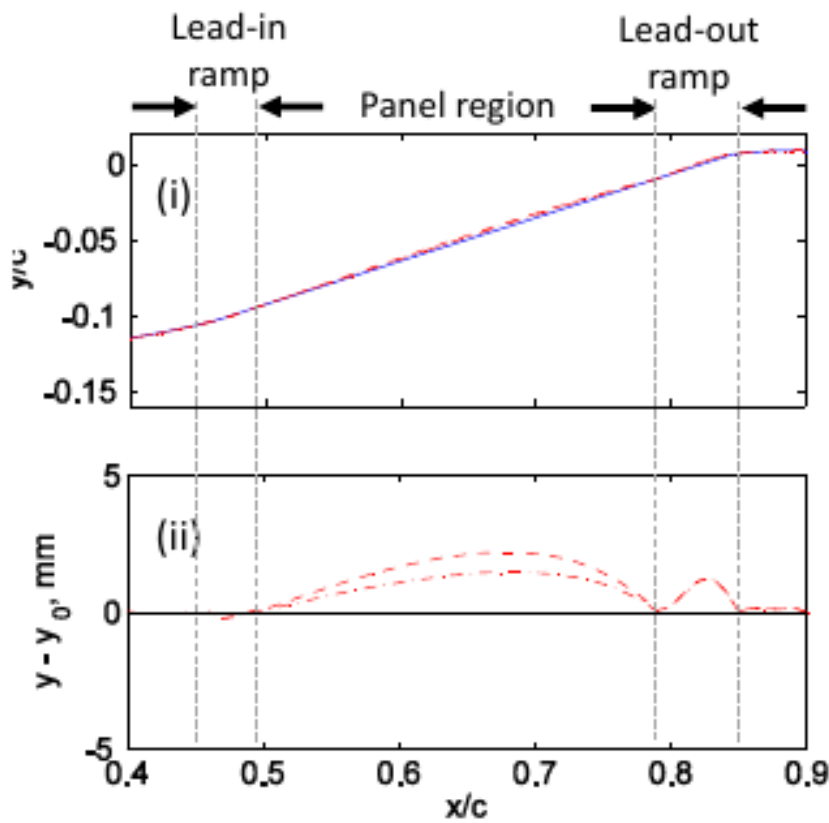

- Modified (rlgld) panels

Modviled (fabrk) pane $1 \mathrm{~s} ; \mathrm{z}=0 \mathrm{~cm}$

Modilled (fabric) panels: $z=6.5 \mathrm{~cm}$

-Modilled (fabrlc) panels, XFOIL: $z=0 \mathrm{~cm}$

Modiried (fabric) panek, XFOIL: $z=6.5 \mathrm{~cm}$ 


\section{Fabric sections: results}

(a)

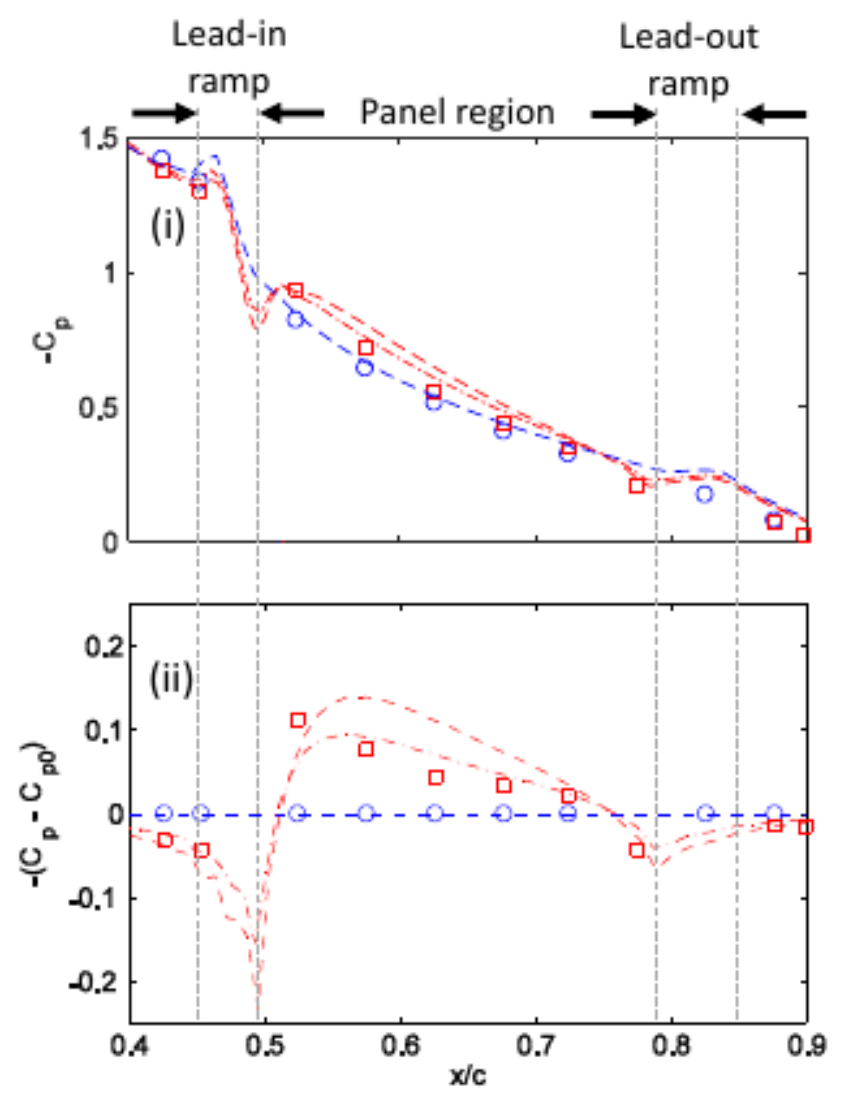

(b)

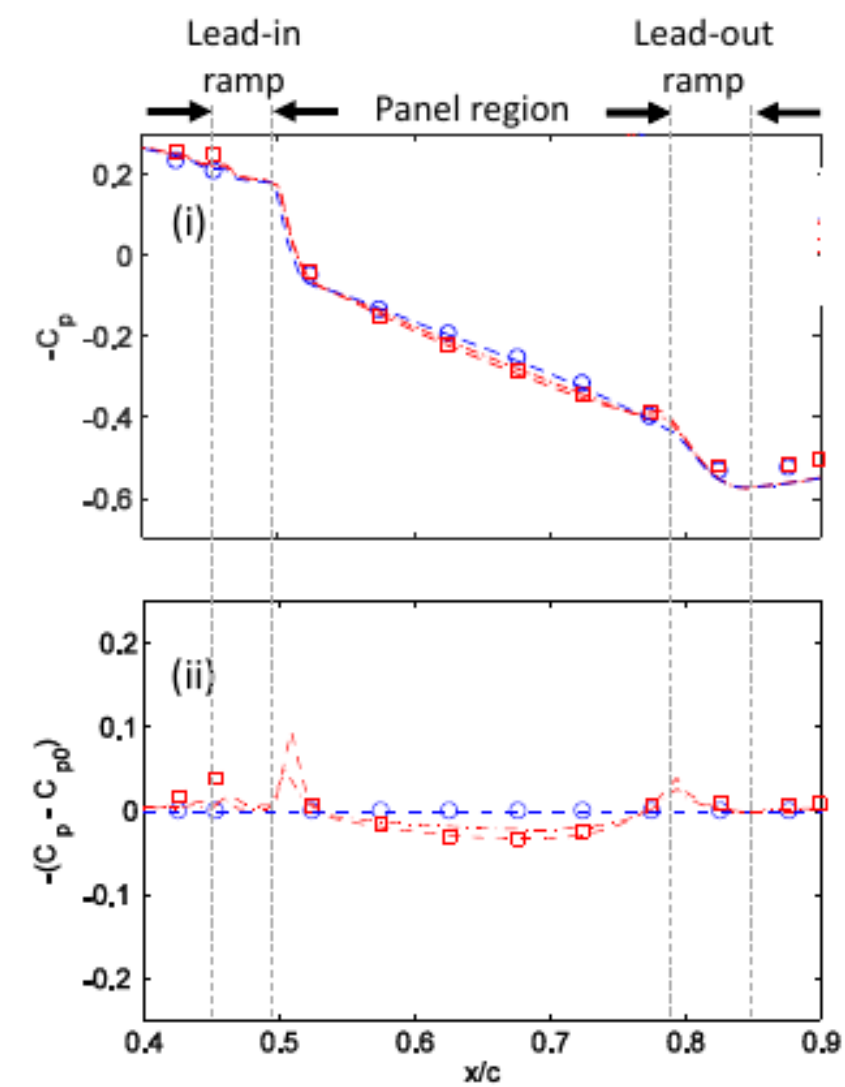




\section{Fabric sections: results}

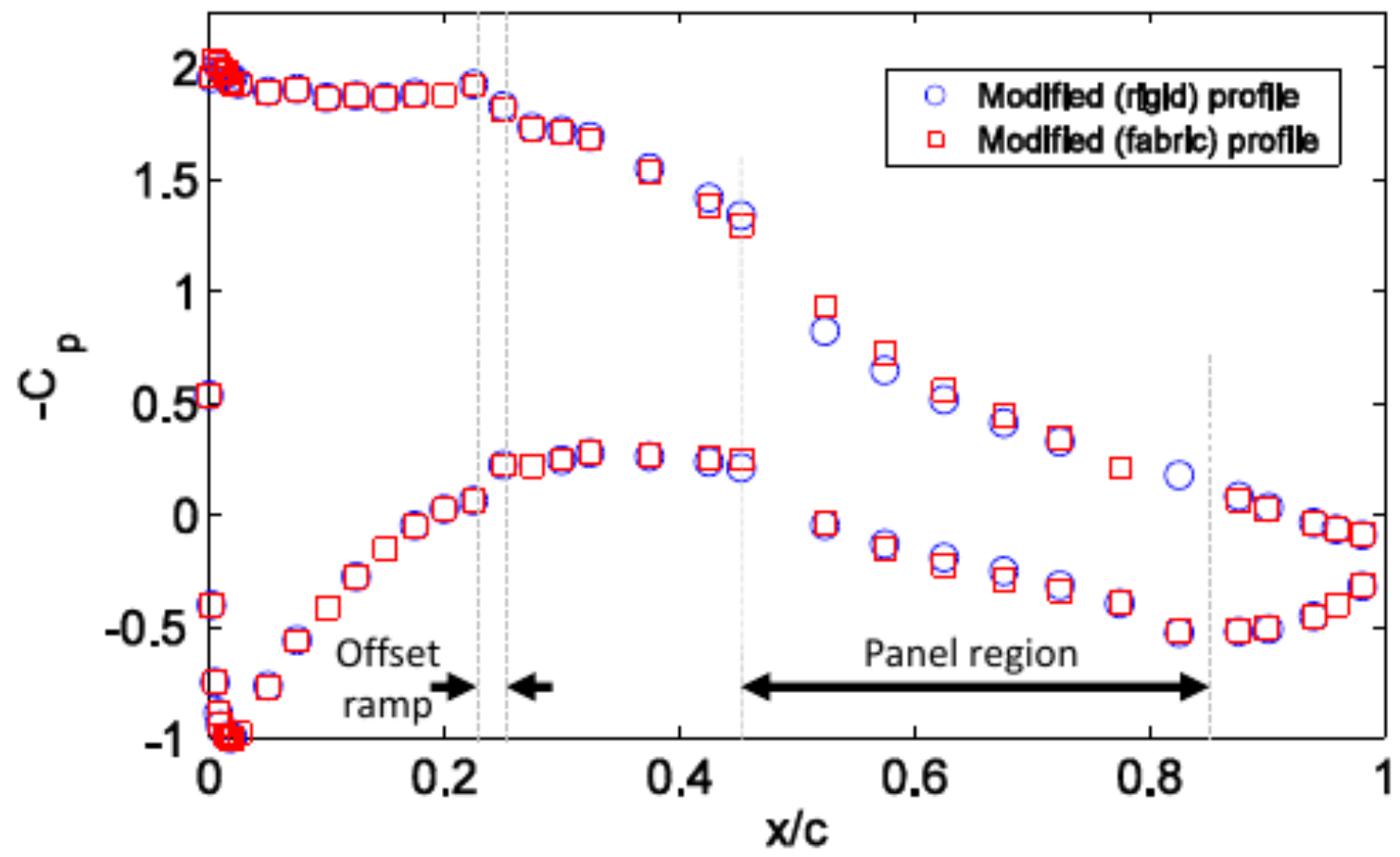




\section{Fabric sections: results}

(a) Flow

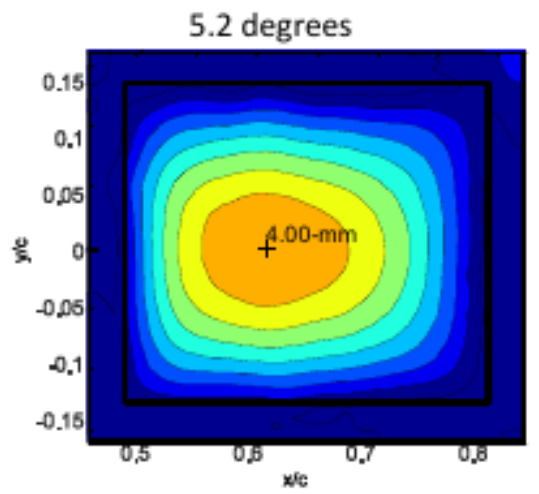

7.3 degrees

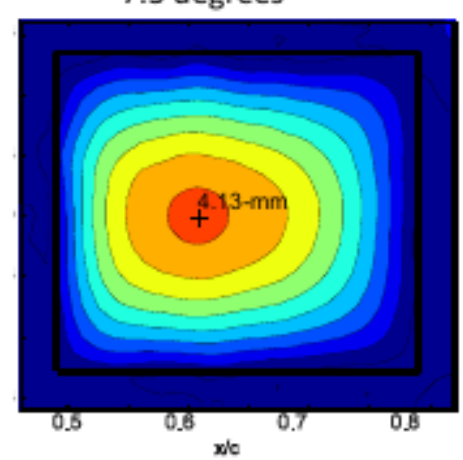

9.2 degrees

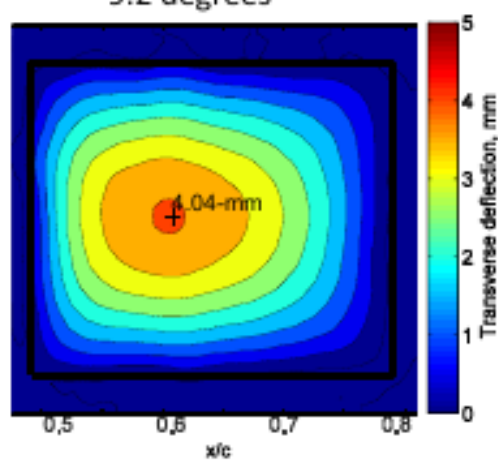

(b)
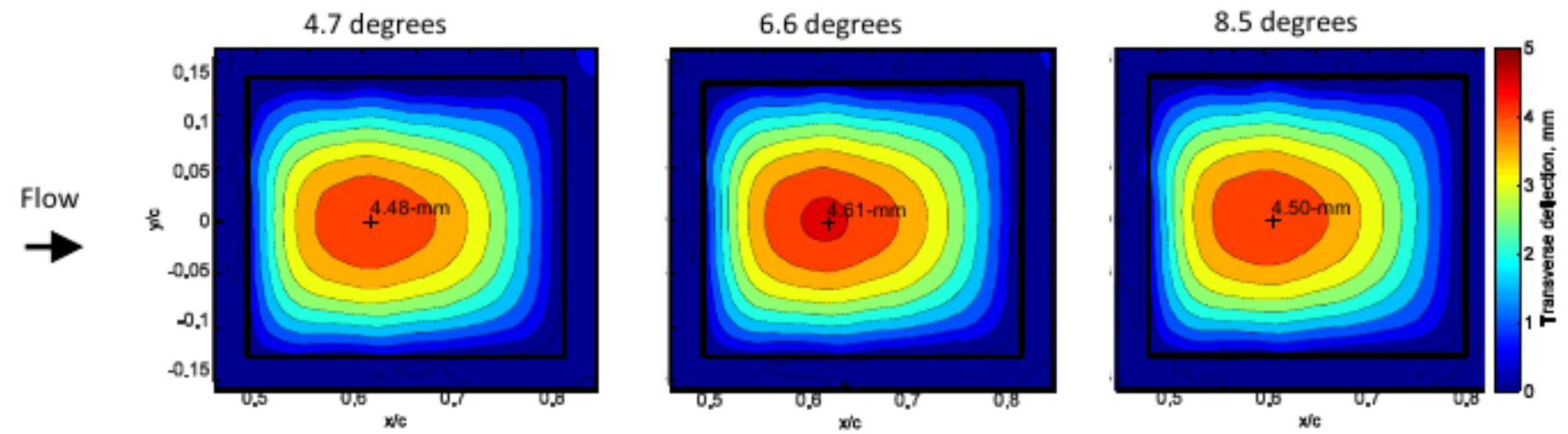


\section{Fabric sections: results}

$4.7 \mathrm{deg}$. [XFOIL: $4.5 \mathrm{deg}$.

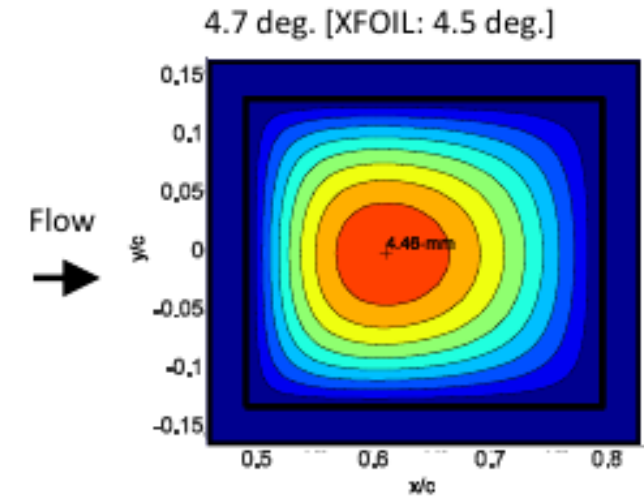

4.7 deg. [XFOIL: 4.5 deg.]

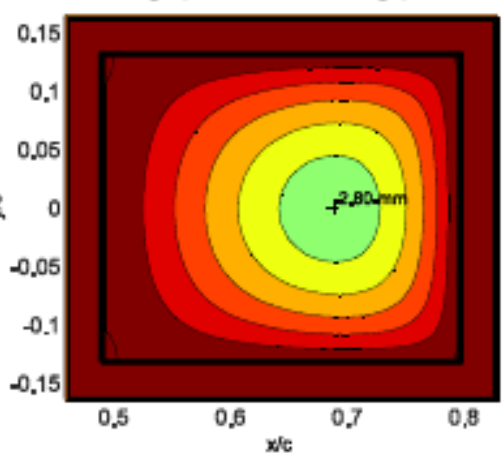

$6.6 \mathrm{deg}$. [XFOIL: $6.4 \mathrm{deg}$.]

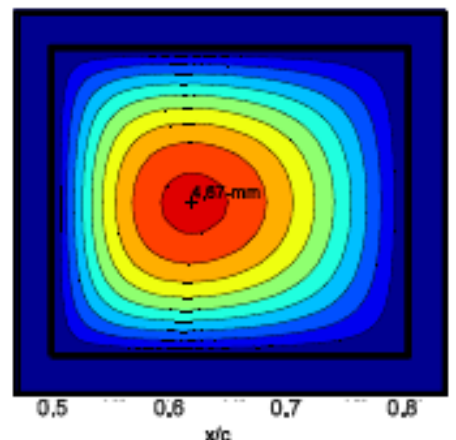

$x / c$

6.6 deg. [XFOIL: 6.4 deg.]

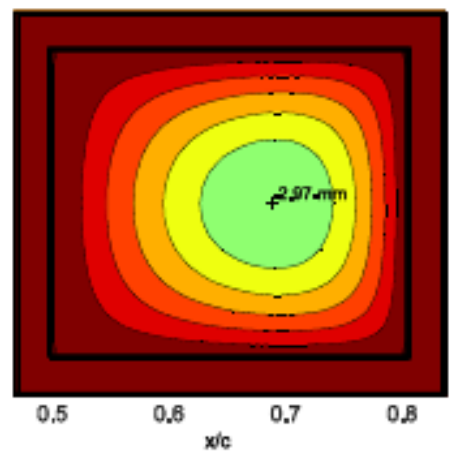

$8.5 \mathrm{deg}$. [XFOIL: $8.0 \mathrm{deg}$.

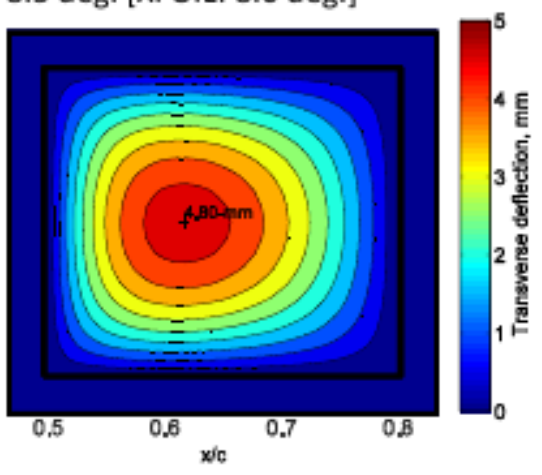

$8.5 \mathrm{deg}$. [XFOIL: $8.0 \mathrm{deg}$.

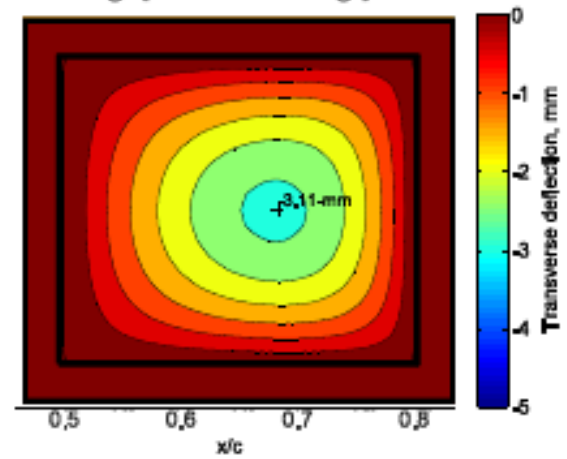




\section{Fabric sections: results}

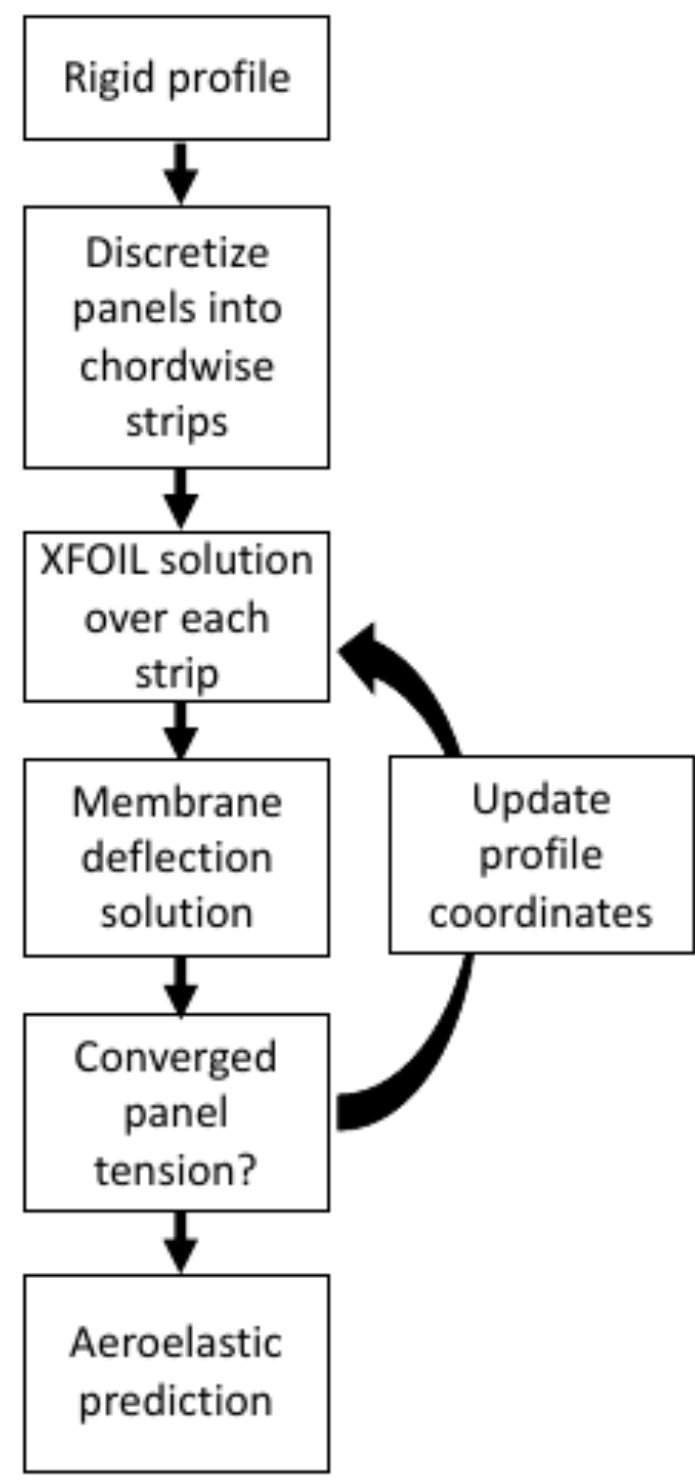




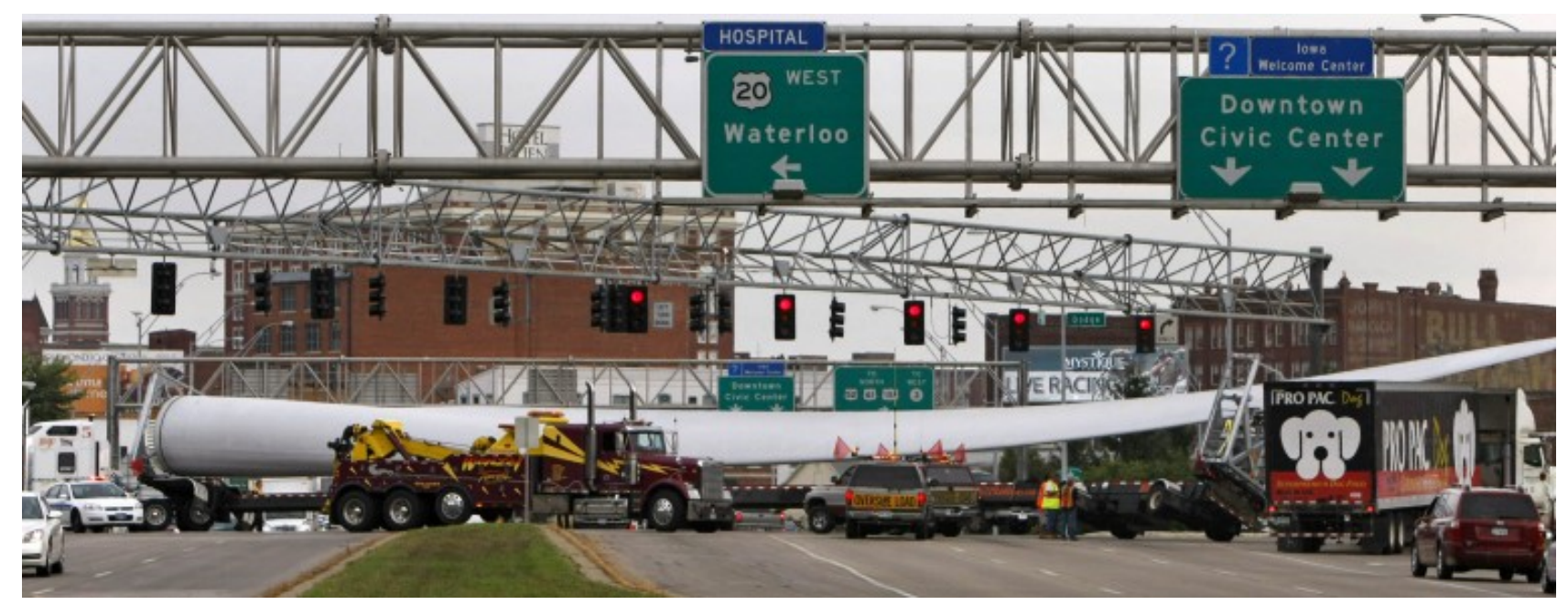

http://www.thonline.com/news/breaking/ article_fe7deb6cc8d5-11e0-9ab6-001a4bcf6878.html 